Engineering Journal

Elsevier Editorial System(tm) for Chemical

Manuscript Draft

Manuscript Number: CEJ-D-18-07067R1

Title: From semi-batch to continuous tubular reactors: a kinetics-free approach

Article Type: Research Paper

Keywords: semi-batch to continuous; process intensification; kineticsfree; tubular reactor

Corresponding Author: Professor renato rota,

Corresponding Author's Institution:

First Author: Federico Florit

Order of Authors: Federico Florit; Valentina Busini; Giuseppe Storti; renato rota

Abstract: A methodology, which does not require any kinetic information, for the rigorous transformation of an isothermal, homogeneous semi-batch process into an equivalent continuous side-fed tubular reactor was developed. Once the semi-batch process parameters are known, the proposed methodology allows for easily defining all the process parameters of a side-fed tubular reactor that guarantees the same performances as the original semi-batch process, in terms of conversion and product characteristics. Two different case studies were selected to investigate the potential of the proposed approach: a copolymer synthesis and the production of a fine chemical, clearly showing the need of a rigorous transformation of the semi-batch process into the continuous one since productivity and product quality are strongly affected by the feeding policy.

Response to Reviewers: Detailed reply to the Reviewers' comments to the manuscript: From semi-batch to continuous tubular reactors: a kineticsfree approach by F. Florit, V. Busini, G. Storti, and R. Rota Manuscript ID: CEJ-D-18-07067

For the Reader's convenience, the original Reviewers' comments are reported followed by our detailed replies.

Reviewer \#1:

I support publication of this paper in "Chemical Engineering Journal". As well known, the kinetic information obtained from the batch reactor can be directly used for describing the steady-state plug-flow reactor. In the paper the next step is done: the kinetic information obtained from the semi-batch reactor can be tranformed into the description of the steady-state tubular reactor with side injections. I consider it is a good result.

I recommend authors to formulate an explicit conclusion on the optimal side feed policy. Generally, does this optimal policy exist? 
If existing, the "optimal" side feed policy can be defined as the policy allowing the tubular reactor to produce a product with exactly the same specifics as that obtained using the SBR recipe. A sentence has been added in the Conclusion section to highlight this concept.

Reviewer \#2:

This is an interesting paper which deals with the specification of reaction conditions for a continuous tubular reactor, based on predefined reaction conditions of a semi-batch reactor (SBR). In principle, this methodology would allow maintaining similar process and product specifications in both SBR and continuous tubular reactor. This study gain relevance given the current industrial need to shift from SBR to continuous tubular reactors, in order to enhance intensification. I consider the paper has an acceptable level of new results, with appropriate length and could be considered for publication at CEJ, only after (minor) revisions are addressed.

-The authors emphasize that the proposed methodology does not require any kinetic information. However, this is not a surprising outcome of the methodology, since the mathematical treatment is forced to have the same species mass fraction in the SBR and the continuous tubular reactor at every corresponding reaction time, by introducing the necessary amount of reactant on the side feeds, in order to establish the similarity in both reactor types. Yet, the authors make use of kinetic treatments for the analysis of their example of copolymerization reaction, which provide much more information that the proposed methodology. For the former case, very well-established relationships for the copolymer composition control have been derived, in terms of reaction kinetics. The authors should highlight the benefits and drawbacks of getting rid of kinetic information for the shift from SBR to continuous tubular reactors.

Even if the proposed methodology does not require any kinetic information, we agree with the Reviewer that kinetic information (when available) allow optimizing the SBR recipe and, consequently, the continuous tubular reactor. A sentence has been added in the conclusion section to highlight this concept. Note that the primary focus of the work is the transfer of a recipe applied in SBR to a continuous tubular reactor while ensuring a final product with the same features: therefore, the achievement of this target without any kinetic information is a major advantage.

-The general methodology is derived for the application to a continuous tubular reactor in which the side-feeds could be situated at continuous locations along the reactor. In such a general case, these equations would be only applicable to a membrane reactor, still with the difficulty of controlling the side feed to the values calculated by the method. Can the authors provide an example of practical application of a reactor with side-injections that are continuous and adjustable along the reactor length, or a methodology for achieving this requisite?

For the sake of example we can mention a coaxial tubular reactor with the co-reactant fed to the outer pipe through the perforated inner pipe: by changing properly the holes' diameter and number, almost any side feed policy can be implemented. However, such a reactor requires a dedicated design procedure, most probably CFD based; work is in progress on this topic, which will be presented in a future paper. 
In section 3, the authors managed to apply their methodology for the transformation of operating conditions for a SBR to a continuous tubular reactor with discrete lateral injections. Figure 6 shows the effect of increasing the number of discrete side-injection points (Nd) on the relative difference of between outlet flowrates for chemical species. It compares the relative error when $\mathrm{Nd}=3$ and $\mathrm{Nd}=5$. It would be rather useful to see a bigger variation in Nd. Furthermore, it would be important to show how the relative error corresponding to the case of discrete lateral injections approaches to the one calculated with continuous injections, when Nd tends to infinity. A critical value for Nd at which the discrete and continuous injection mode behave similarly could be found.

We agree with the Reviewer and the new Figure 7 has been added showing the required information.

Example 4.2 make use of several continuous tubular reactors for the study, but still each tubular reactor is analyzed with continuous side injections.

Maybe there was a misunderstanding: example 4.2 is actually based on a single tubular continuous reactor with multiple side feedings; in order to make this point more clear we have partly rephrased the third paragraph of the section.

-Equation (27) seems to have a typo. The dimensionless feed rate is not dimensionless. The total time $t$ proc should multiply both terms on the right-hand sides of the equation.

We thank the Reviewer for her/his kind correction, which has been introduced in the paper.

-In Figures 3, 4 and 6, it would be helpful for the reader to have a legend with the meaning of the values for the parameters employed (For example, in Figure 6a, instead of simply labeling the first curve with 1,1 , better add $\left.k 1=1 e-6 \mathrm{~m}^{\wedge} 3 \mathrm{kmol}^{\wedge}-1 \mathrm{~s}^{\wedge}-1, \mathrm{k} 2=1 \mathrm{e}-4 \mathrm{~s}^{\wedge}-1\right)$.

The Figures were modified according to the Reviewer's suggestion.

-Most of the figures presented to analyze the methodology compare feed rates along the reactor length. In order to validate the methodology, it would be useful to compare mass fraction of chemical species in the SBR and the corresponding continuous tubular reactor to show that effectively, similar mass fractions are preserved. This would be particularly important when discrete side injections are employed.

With a continuous side feed, the mass fraction of chemical species in time (for the SBR) and in residence time (for the tubular reactor) are superimposed, therefore making such a comparison not really meaningful. However, we agree with the Reviewer that it is useful comparing the species profile when a discrete side feed policy is used. Therefore, the new Figure 8 compares (for a specific condition) the mass fraction profile of species $C$ for the SBR with those of the tubular reactor with three different numbers of side injections. 
The manuscript treats the general conversion of a semi-batch reactor (SBR) into an equivalent continuous side-fed tubular reactor (PFR)

operating in the steady state. In addition to the general equations, two examples are considered, production of a copolymer and of a diol via a consecutive reaction.

In general, this is a good paper. It is written well and is publishable after accounting for the following comments.

1. It is claimed that the methodology is new (first sentence of Abstract). While it is true that the general case is considered here, and I'm unsure if this has been done before, it's a stretch to claim that the methodology is new. The equivalence of a SBR with a continuous sidefed PFR is given in standard CRE textbooks.

We agree with the Reviewer that the conceptual equivalence is well established, even if in the Authors knowledge it has never been formalized in a general mathematical methodology. However, to avoid misunderstandings, we have removed the terms "new" from the Abstract and "novel" from the Conclusion section.

2. Since the material is relatively straightforward, I think that a reduction in length of the manuscript is justifiable and possible. I expect that without compromising understanding or readability, one could achieve a reduction in length by about $25 \%$

Even if the mathematical derivation could be considered relatively straightforward, it would be really hard making the methodology derivation understandable with a strong reduction of the paper length. The only exceptions are the original Figures 7 and 8 that have been eliminated without compromising the paper readability. However, since Reviewer 2 not only considered the length of the paper "appropriate", but also required some additional information to be added to the manuscript, the elimination of the two aforementioned figures did not result in a significant paper shortage since two new figures have been added as required by Reviewer 2 . 
POLITECNICO

MILANO 1863

Milano, 06 August 2018

DIPARTIMENTO DI

CHIMICA, MATERIALI E

INGEGNERIA CHIMICA

"GIULIO NATTA"

Renato Rota

Dear Editor

Please find enclosed a revised version of the manuscript From semi-batch to continuous tubular reactors: a kineticsfree approach by Florit et al. (Manuscript ID: CEJ-D-1807067), which we submit for publication in Chemical Engineering Journal.

I believe that we have satisfactory accounted for all the comments of the Reviewers, as detailed in the attached list.

Best regards

Renato Rota

Professor 


\section{*List of Suggested Reviewers}

Prof. Giuseppe Maschio

Dipartimento di Ingegneria Industriale

Università di Padova

VIA F.MARZOLO, 9

35122 PADOVA, Italy

E-mail: giuseppe.maschio@unipd.it

Prof. Rosa Nomen

Institut Quimic de Sarria

Ramon Llul University

43 Avenir

E-08021 Barcelona, Spain

Email: rnome@iqs.edu

Prof. Arvind Varma

Purdue University

School of Chemical Engineering

Forney Hall of Chemical Engineering

480 Stadium Mall Drive

West Lafayette, IN 47907-2100

E-mail: avarma@ecn.purdue.edu 
Detailed reply to the Reviewers' comments to the manuscript: From semi-batch to continuous tubular reactors: a kinetics-free approach by F. Florit, V. Busini, G. Storti, and R. Rota

\section{Manuscript ID: CEJ-D-18-07067}

For the Reader's convenience, the original Reviewers' comments are reported in italic followed by our detailed replies.

\section{Reviewer \#1:}

I support publication of this paper in "Chemical Engineering Journal". As well known, the kinetic information obtained from the batch reactor can be directly used for describing the steady-state plug-flow reactor. In the paper the next step is done: the kinetic information obtained from the semi-batch reactor can be tranformed into the description of the steady-state tubular reactor with side injections. I consider it is a good result.

I recommend authors to formulate an explicit conclusion on the optimal side feed policy. Generally, does this optimal policy exist?

If existing, the "optimal" side feed policy can be defined as the policy allowing the tubular reactor to produce a product with exactly the same specifics as that obtained using the SBR recipe. A sentence has been added in the Conclusion section to highlight this concept. 


\section{Reviewer \#2:}

This is an interesting paper which deals with the specification of reaction conditions for a continuous tubular reactor, based on pre-defined reaction conditions of a semi-batch reactor (SBR). In principle, this methodology would allow maintaining similar process and product specifications in both SBR and continuous tubular reactor. This study gain relevance given the current industrial need to shift from SBR to continuous tubular reactors, in order to enhance intensification.

I consider the paper has an acceptable level of new results, with appropriate length and could be considered for publication at CEJ, only after (minor) revisions are addressed.

-The authors emphasize that the proposed methodology does not require any kinetic information. However, this is not a surprising outcome of the methodology, since the mathematical treatment is forced to have the same species mass fraction in the SBR and the continuous tubular reactor at every corresponding reaction time, by introducing the necessary amount of reactant on the side feeds, in order to establish the similarity in both reactor types. Yet, the authors make use of kinetic treatments for the analysis of their example of copolymerization reaction, which provide much more information that the proposed methodology. For the former case, very well-established relationships for the copolymer composition control have been derived, in terms of reaction kinetics. The authors should highlight the benefits and drawbacks of getting rid of kinetic information for the shift from SBR to continuous tubular reactors.

Even if the proposed methodology does not require any kinetic information, we agree with the Reviewer that kinetic information (when available) allow optimizing the SBR recipe and, consequently, the continuous tubular reactor. A sentence has been added in the Conclusion section to highlight this concept. Note that the primary focus of the work is the transfer of a recipe applied in SBR to a continuous tubular reactor while ensuring a final product with the same features: therefore, the achievement of this target without any kinetic information is a major advantage.

-The general methodology is derived for the application to a continuous tubular reactor in which the sidefeeds could be situated at continuous locations along the reactor. In such a general case, these equations would be only applicable to a membrane reactor, still with the difficulty of controlling the side feed to the values calculated by the method. Can the authors provide an example of practical application of a reactor with side-injections that are continuous and adjustable along the reactor length, or a methodology for achieving this requisite?

For the sake of example we can mention a coaxial tubular reactor with the co-reactant fed to the outer pipe through the perforated inner pipe: by changing properly the holes' diameter and number, almost any side feed policy can be implemented. However, such a reactor requires a dedicated design procedure, most probably CFD based; work is in progress on this topic, which will be presented in a future paper.

In section 3, the authors managed to apply their methodology for the transformation of operating conditions for a SBR to a continuous tubular reactor with discrete lateral injections. Figure 6 shows the effect of increasing the number of discrete side-injection points $(\mathrm{Nd})$ on the relative difference of between 
outlet flowrates for chemical species. It compares the relative error when $N d=3$ and $N d=5$. It would be rather useful to see a bigger variation in Nd. Furthermore, it would be important to show how the relative error corresponding to the case of discrete lateral injections approaches to the one calculated with continuous injections, when Nd tends to infinity. A critical value for $\mathrm{Nd}$ at which the discrete and continuous injection mode behave similarly could be found.

We agree with the Reviewer and the new Figure 7 has been added showing the required information.

Example 4.2 make use of several continuous tubular reactors for the study, but still each tubular reactor is analyzed with continuous side injections.

Maybe there was a misunderstanding: example 4.2 is actually based on a single tubular continuous reactor with multiple side feedings; in order to make this point more clear we have partly rephrased the third paragraph of the section.

-Equation (27) seems to have a typo. The dimensionless feed rate is not dimensionless. The total time t_proc should multiply both terms on the right-hand sides of the equation.

We thank the Reviewer for her/his kind correction, which has been introduced in the paper.

-In Figures 3, 4 and 6, it would be helpful for the reader to have a legend with the meaning of the values for the parameters employed (For example, in Figure 6a, instead of simply labeling the first curve with 1,1, better add $\left.k 1=1 e-6 m^{\wedge} 3 k m o / \wedge-1 s^{\wedge}-1, k 2=1 e-4 s^{\wedge}-1\right)$.

The Figures were modified according to the Reviewer's suggestion.

-Most of the figures presented to analyze the methodology compare feed rates along the reactor length. In order to validate the methodology, it would be useful to compare mass fraction of chemical species in the $S B R$ and the corresponding continuous tubular reactor to show that effectively, similar mass fractions are preserved. This would be particularly important when discrete side injections are employed.

With a continuous side feed, the mass fraction of chemical species in time (for the SBR) and in residence time (for the tubular reactor) are superimposed, therefore making such a comparison not really meaningful. However, we agree with the Reviewer that it is useful comparing the species profile when a discrete side feed policy is used. Therefore, the new Figure 8 compares (for a specific condition) the mass fraction profile of species $C$ for the SBR with those of the tubular reactor with three different numbers of side injections. 


\section{Reviewer \#3:}

The manuscript treats the general conversion of a semi-batch reactor (SBR) into an equivalent continuous side-fed tubular reactor (PFR) operating in the steady state. In addition to the general equations, two examples are considered, production of a copolymer and of a diol via a consecutive reaction.

In general, this is a good paper. It is written well and is publishable after accounting for the following comments.

1. It is claimed that the methodology is new (first sentence of Abstract). While it is true that the general case is considered here, and I'm unsure if this has been done before, it's a stretch to claim that the methodology is new. The equivalence of a SBR with a continuous side-fed PFR is given in standard CRE textbooks.

We agree with the Reviewer that the conceptual equivalence is well established, even if in the Authors knowledge it has never been formalized in a general mathematical methodology. However, to avoid misunderstandings, we have removed the terms "new" from the Abstract and "novel" from the Conclusion section.

2. Since the material is relatively straightforward, I think that a reduction in length of the manuscript is justifiable and possible. I expect that without compromising understanding or readability, one could achieve a reduction in length by about $25 \%$.

Even if the mathematical derivation could be considered relatively straightforward, it would be really hard making the methodology derivation understandable with a strong reduction of the paper length. The only exceptions are the original Figures 7 and 8 that have been eliminated without compromising the paper readability. However, since Reviewer 2 not only considered the length of the paper "appropriate", but also required some additional information to be added to the manuscript, the elimination of the two aforementioned figures did not result in a significant paper shortage since two new figures have been added as required by Reviewer 2 . 


\section{Highlights}

- A methodology for transforming a SBR process into a tubular reactor one is proposed

- The tubular reactor involves a properly defined continuous side feed

- The proposed methodology does not require any kinetic information

- The syntheses of a copolymer and a fine chemical are investigated as a case study 


\title{
From semi-batch to continuous tubular reactors: a kinetics-free approach
}

\author{
Federico Florit ${ }^{\mathrm{a}}$, Valentina Busini ${ }^{\mathrm{a}}$, Giuseppe Storti $^{\mathrm{b}}$, Renato Rota ${ }^{\mathrm{a}, *}$ \\ ${ }^{a}$ Politecnico di Milano, Dipartimento di Chimica, Materiali e Ingegneria Chimica G. Natta, via Mancinelli 7, 20131, \\ Milano, Italy \\ ${ }^{b}$ ETH Zürich, Department of Chemistry and Applied Biosciences, Vladimir-Prelog-Weg 1-5/10, HCI F 125, 8093 Zürich, \\ Switzerland
}

\begin{abstract}
A methodology, which does not require any kinetic information, for the rigorous transformation of an isothermal, homogeneous semi-batch process into an equivalent continuous side-fed tubular reactor was developed. Once the semi-batch process parameters are known, the proposed methodology allows for easily defining all the process parameters of a side-fed tubular reactor that guarantees the same performances as the original semi-batch process, in terms of conversion and product characteristics.

Two different case studies were selected to investigate the potential of the proposed approach: a copolymer synthesis and the production of a fine chemical, clearly showing the need of a rigorous transformation of the semi-batch process into the continuous one since productivity and product quality are strongly affected by the feeding policy.
\end{abstract}

Keywords: semi-batch to continuous, process intensification, kinetics-free, tubular reactor

\section{Introduction}

Fine chemicals and specialties are generally produced via discontinuous or semi-continuous processes. This is mainly due either to discontinuous market needs (seasonal campaigns), which require a high degree of flexibility in the synthesis, or to complex and dangerous reaction processes, which are carried out in semi-batch reactors, SBRs, for safety reason [1, 2].

An effective way to reduce costs and enhance reproducibility of such processes is to transform discontinuous ones into their continuous counterpart. This way reactor volumes are usually reduced, the intrinsic process safety is increased thanks to the lower hold-up, and investment costs are also decreased [3]. Moreover, overall process times can be reduced and more constant quality is achieved, with a general decrease in

\footnotetext{
*Corresponding author

Email address: renato.rota@polimi.it (Renato Rota)
} 
operative costs [4]. This is the framework of the so called process intensification. Transition from discontinuous to continuous processes is also motivated by the increasing demand of specialty products. Higher production volumes would require more complex and expensive discontinuous equipment, making engineers more prone to consider continuous systems.

Straightforward choices for continuous reactors are mixed vessels (approximating the behaviour of Continuous Stirred Tank Reactors, CSTRs) [5] or tubular reactors (aiming at Plug Flow Reactors, PFRs) [3]. This last configuration also benefits better heat exchange due to higher heat transfer area/volume ratios [6]; as a consequence, it is possible to run reactions in more severe conditions leading to an increase in product quantity/quality, while keeping the process safe. Mixed vessels for continuous synthesis are well investigated and find several industrial applications. On the contrary, only few works analyse the same problem in tubular reactors. Roughly speaking, four main research areas for continuous syntheses can be identified: classical PFR-like reactors [6, 7], modular systems [8-11], microreactors [12] and other reactors [13].

Ferrouillant et al. [6] have studied the improved heat removal in a multifunctional heat exchanger running an exothermic reaction. Rossow et al. [7] have designed a continuous copolymerisation PFR-like reactor from a process carried out in a SBR. Kohlmann et al. [8], Goerke et al. [9] and Hashemi et al. [11] have developed a continuous modular tubular reactor with intermediate injections for a copolymerisation reaction, analysing its feasibility with respect to the original SBR process and proposing also an optimised control system. Meimaroglou et al. [10] used a series of tubular reactors in a modular system to run a solution copolymerisation varying process conditions. Haber et al. [12] have analysed the behaviour of a microstructured tubular reactor running a quasi-instantaneous exothermic reaction. Anxionnaz et al. [13] reviewed different configurations and geometries for process intensification in heat exchanger reactors. All previous works succeeded in finding a specific condition for carrying out the desired process in a given reactor, but seem to lack a sense of generality, being the reported approach limited to the particular process under examination.

This work focuses on continuous side-fed tubular reactors. In particular a PFR with continuous side injections, similar to the one proposed by Zwietering [14], was considered. Given a reaction process effectively carried out in a semi-batch reactor with an optimised recipe, the primary aim was to define the feeding policy for the side injections into the Zwietering-like tubular reactor which allows to reproduce exactly the SBR behaviour without knowing the reaction kinetics. This way, the operating conditions of a tubular reactor having the same efficiency as the original SBR in terms of conversion and selectivity are identified. On the 
other hand, the process productivity remains as degree of freedom and, therefore, can be increased.

\section{Model formulation}

\subsection{Dimensional equations}

Let us consider a generic industrial reaction process through which a chemical species is produced using an optimised recipe in SBR. Figure 1 shows the scheme of such SBR, which is initially charged with a known quantity of each species, $m_{i, 0}$, and fed with a mass flowrate of each component, $F_{i}(t),(i=1, \ldots, N C)$. The reactor is assumed to be well mixed and to operate at isothermal conditions.

Our aim is to transform this discontinuous process into a continuous, steady-state one, using a tubular reactor with side feed injections (Figure 2) and constant temperature (equal to that of the SBR). The key question to be answered is how to distribute the side feed injections to obtain the same performances (in terms of conversion and selectivity) as the original industrial process carried out in SBR.

\subsubsection{SBR governing equations}

The original SBR can be modelled using the conventional conservation equations, considering a process duration equal to $t_{\text {proc }}$. The mass balance equations in terms of mass fractions are:

$$
\frac{d \omega_{i}^{*}}{d t}=\frac{F_{i}(t)}{m}-\omega_{i}^{*} \frac{F(t)}{m}+\frac{\dot{\Omega}_{i}\left(\underline{\omega}^{*}\right)}{\rho^{*}(t)}
$$

where $\omega_{i}^{*}$ is the mass fraction of species $i$ in the SBR, $t$ is the time, $m$ is the total mass, $\dot{\Omega}_{i}$ is species $i$ massive reaction rate, and $\rho^{*}$ is the density of the reactive mixture.

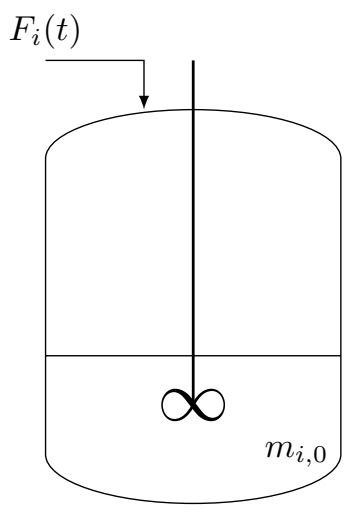

Figure 1: Semi-batch reactor. $m_{i, 0}[\mathrm{~kg}]$ is the initially charged amount of species $i, F_{i}(t)\left[\mathrm{kg} \mathrm{s}^{-1}\right]$ is the feed rate of species $i$. 


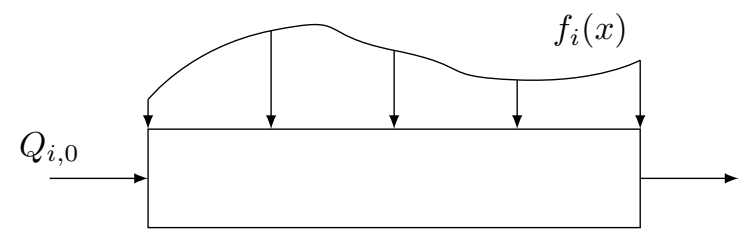

Figure 2: Tubular reactor with distributed side feed. $Q_{i, 0}\left[\mathrm{~kg} \mathrm{~s}^{-1}\right]$ is the inlet flow rate of species $i, f_{i}(x)\left[\mathrm{kg} \mathrm{m}^{-1} \mathrm{~s}^{-1}\right]$ is the specific side feed rate of species $i$.

An additional equation (the overall mass balance) is required to track the total mass increase in time:

$$
\frac{d m}{d t}=F(t)
$$

where

$$
F(t) \stackrel{\text { def }}{=} \sum_{i=1}^{N C} F_{i}(t)
$$

This system of ODEs is completed by the corresponding initial conditions, ICs:

$$
\begin{gathered}
\omega_{i}^{*}(0)=\frac{m_{i, 0}}{m_{0}} \\
m(0)=m_{0}
\end{gathered}
$$

where $m_{0}=\sum_{i=1}^{N C} m_{i, 0}$ is the total initial mass.

Note that the system density, $\rho^{*}$, can be computed through a proper equation of state as a function of composition (and therefore time):

$$
\rho^{*}(t)=\rho\left(\underline{\omega}^{*}(t)\right)
$$

The simplest equation of state that can be used is obviously $\rho^{*}(t)=$ const.

The equations above are used to compute the time evolution of mass fractions, $\omega_{i}^{*}$, total mass, $m$, and overall density, $\rho^{*}$, given the reaction kinetics, $\dot{\Omega}_{i}\left(\underline{\omega}^{*}\right)$, and the feed rates, $F_{i}(t)$. However, it is worth noting that also measurements of the time evolutions of the same quantities (composition, overall mass, and density) in the existing industrial SBR are enough to use the proposed approach.

\subsubsection{Tubular reactor governing equations}

Similar mass balance equations can be written for the tubular reactor with side injections at steady state:

$$
\frac{d \omega_{i}}{d x}=\frac{1}{Q}\left(f_{i}(x)-\omega_{i} f(x)+\dot{\Omega}_{i}(\underline{\omega}) \mathscr{A}(x)\right)
$$


where $\omega_{i}$ is species $i$ mass fraction in the tubular reactor, $x$ is the axial coordinate, $Q$ is the total mass flowrate, $\mathscr{A}$ is the cross sectional area and

$$
f(x) \stackrel{\text { def }}{=} \sum_{i=1}^{N C} f_{i}(x)
$$

The total flowrate changes along the axial coordinate according to the overall mass balance:

$$
\frac{d Q}{d x}=f(x)
$$

Again, the previous equations ask for boundary conditions, BCs:

$$
\begin{gathered}
\omega_{i}(0)=\omega_{i, 0} \\
Q(0)=Q_{0}
\end{gathered}
$$

where $Q_{0}$ and $\omega_{i, 0}$ are the inlet mass flowrate and inlet species $i$ mass fraction, respectively.

Again, an equation of state (the same used to simulate the SBR) is required to evaluate the density profile, $\rho(x)$, as a function of composition:

$$
\rho(x)=\rho(\underline{\omega}(x))
$$

Finally, it is convenient to introduce the elapsed time, $\theta(x)$, defined as the time spent by a generic fluid element to reach the position $x$ after entering the reactor:

$$
\frac{d \theta}{d x}=\frac{\rho(x) \mathscr{A}(x)}{Q(x)}
$$

This equation follows from the definition of the fluid parcel instantaneous velocity from an inertial observer point of view. It also provides the connection between time and space inside the tubular reactor and it will be helpful to build the relationship between the SBR and the Zwietering-like reactor.

The final system involves $(N C+4)$ equations (namely, Equations (7), (8), (9), (12) and (13)) and $(3 N C+7)$ unknowns: $\underline{\omega}(x), \underline{\omega}_{0}, Q(x), Q_{0}, \underline{f}(x), f(x), \theta(x), \rho(x), \mathscr{A}$, and the reactor length $L$. Therefore, $(2 N C+3)$ degrees of freedom need to be specified to make the problem defined. 


\subsubsection{Proposed semi-batch to continuous transformation procedure}

A connection between the SBR and a Zwietering-like reactor can be established forcing the same species mass fraction to be present at a given SBR time $(t)$ and at the corresponding tubular reactor position $(x)$, that is, at the coordinate reached by a fluid element after time $t=\theta(x)$ :

$$
\omega_{i}(x)=\omega_{i}^{*}(\theta(x)) \quad \forall x \in[0, L]
$$

Note that, as a consequence, the same relation holds true for the density, i.e., $\rho(x)=\rho^{*}(\theta(x))$.

Moreover, to ensure the same overall process time in both reactors, the following equation applies:

$$
\theta(L)=t_{\text {proc }}
$$

Equations (14) and (15) enforce $2 N C+1$ additional constraints, being the former valid not only along the reactor, but also at the inlet where the BCs (10) apply (i.e., $\left.\omega_{i}(0)=\omega_{i}^{*}(0)=\frac{m_{i, 0}}{m_{0}}\right)$. Therefore, 2 degrees of freedom remain, which can be saturated by setting arbitrary values of $Q_{0}$ and $\mathscr{A}(x)$. Note that different values of these quantities lead to different values of productivity and length of the tubular reactor.

Equations (7) and (14) together with (13) lead to (note that in the following the dependencies on $x$ are dropped for brevity):

$$
\omega_{i}^{*}(\theta) f+\left.Q \frac{d \omega_{i}^{*}}{d \theta}\right|_{\theta=t} \frac{d \theta}{d x}=\omega_{i}^{*}(\theta) f+\left.\frac{d \omega_{i}^{*}}{d t}\right|_{t=\theta} \rho \mathscr{A}=f_{i}+\dot{\Omega}_{i}\left(\underline{\omega}^{*}(\theta)\right) \mathscr{A}
$$

which, combined with Equation (1), becomes:

$$
\omega_{i}^{*}(\theta)\left(f-\rho \mathscr{A} \frac{F(\theta)}{m(\theta)}\right)=\omega_{i}^{*}(\theta) \sum_{j=1}^{N C}\left(f_{j}-\rho \mathscr{A} \frac{F_{j}(\theta)}{m(\theta)}\right)=f_{i}-\rho \mathscr{A} \frac{F_{i}(\theta)}{m(\theta)}
$$

where, $m(\theta)$ can be obtained from Equation (2) as:

$$
m(\theta)=m_{0}+\int_{0}^{\theta} F(t) d t
$$


Equation (17) leads to a linear system $\underline{\underline{A} y}=\underline{0}$, where

$$
\begin{aligned}
\underline{\underline{A}}= & {\left.\left[\begin{array}{cccc}
\omega_{1}^{*} & \omega_{1}^{*} & \ldots & \omega_{1}^{*} \\
\omega_{2}^{*} & \omega_{2}^{*} & \ldots & \omega_{2}^{*} \\
\vdots & \vdots & \ddots & \vdots \\
\omega_{N C}^{*} & \omega_{N C}^{*} & \ldots & \omega_{N C}^{*}
\end{array}\right]\right|_{t=\theta}-\underline{\underline{I}} } \\
\underline{y} & =\left[\begin{array}{c}
f_{1}-\rho \mathscr{A} \frac{F_{1}(\theta)}{m(\theta)} \\
f_{2}-\rho \mathscr{A} \frac{F_{2}(\theta)}{m(\theta)} \\
\vdots \\
f_{N C}-\rho \mathscr{A} \frac{F_{N C}(\theta)}{m(\theta)}
\end{array}\right]
\end{aligned}
$$

and $\underline{\underline{I}}$ is the identity matrix. Nevertheless, these equations are not linearly independent. In fact, an identity is obtained if all equations are summed up since the stoichiometric condition $\sum_{i=1}^{N C} \omega_{i}^{*}=1$ holds true. It should be noted that at least one unknown is zero: this is the case when a species is neither fed to the SBR nor to the tubular reactor side (for instance, this is true when the species is a product). In this case the solution of the system is the trivial one, $\underline{y}=\underline{0}$, that is:

$$
f_{i}-\rho \mathscr{A} \frac{F_{i}(\theta)}{m(\theta)}=0
$$

The same solution could be obtained even if all species were fed to the SBR since one function $f_{k}(x)$ can be arbitrarily chosen to fulfil the constraint $y_{k}=0$. Therefore, in any case it follows that the solution of the previous linear system of equations reduces to:

$$
f_{i}(x)=\rho \mathscr{A} \frac{F_{i}(\theta(x))}{m(\theta(x))}
$$

This last equation can be recast as:

$$
\frac{f_{i}(x)}{\mathscr{A}(x)}=\frac{F_{i}(\theta(x))}{V(\theta(x))}
$$

where $V(\theta(x))=\frac{m(\theta(x))}{\rho^{*}(\theta(x))}$ is the reaction volume in the SBR at time $t=\theta(x)$. This means that the volume specific mass flowrate must be the same in both the SBR and the Zwietering-like reactor at the same time. Note that the ratio $f_{i}(x)$ to $\mathscr{A}(x)$ has units of flowrate per volume and it represents the feed profile which allows reproducing a process carried out in a SBR by a continuous tubular reactor while keeping the same performances in terms of both conversion and selectivity. 


\subsubsection{Model constitutive equations}

The proposed model for the rigorous transformation of a SBR process into a Zwietering-like tubular reactor process is based on Equations (9), (13), (15) and (21) together with the required boundary conditions, that is:

$$
\left\{\begin{array} { l } 
{ \frac { d Q } { d x } = \sum _ { i = 1 } ^ { N C } f _ { i } ( x ) } \\
{ \frac { d \theta } { d x } = \frac { \rho \mathscr { A } } { Q ( x ) } } \\
{ \theta ( L ) = t _ { \text { proc } } } \\
{ f _ { i } ( x ) = \rho \mathscr { A } \frac { F _ { i } ( \theta ) } { m ( \theta ) } }
\end{array} \quad \left\{\begin{array}{l}
\theta(0)=0 \\
Q(0)=Q_{0}
\end{array}\right.\right.
$$

This is a set of 2 ODEs with Dirichlet BCs and $N C+1$ algebraic relationships. Note that the algebraic equations are uncoupled from the ODEs solution, therefore this system is not DAE. From a numerical point of view, the ODE system must be integrated until the exit condition, $\theta(L)=t_{\text {proc }}$, is met, thus providing the tubular reactor length. Moreover, since the ODEs solution provides the $\theta(x)$ relation, the required side feed rates $f_{i}(x)$ can be easily computed.

\subsection{Dimensionless equations}

Dimensionless equations can be obtained from the aforementioned ones by defining the following dimensionless variables:

$$
\begin{aligned}
& \hat{x} \stackrel{\text { def }}{=} \frac{x}{L} \rightarrow \quad d x=L d \hat{x} \\
& \hat{t} \stackrel{\text { def }}{=} \frac{t}{t_{\text {proc }}} \rightarrow d t=t_{\text {proc }} d \hat{t} \\
& \hat{Q}(\hat{x}) \stackrel{\text { deff }}{=} \frac{Q(L \hat{x})}{Q_{0}} \rightarrow d Q=Q_{0} d \hat{Q} \\
& \hat{f}_{i}(\hat{x}) \stackrel{\text { def }}{=} \frac{L}{Q_{0}} f_{i}(L \hat{x}) \\
& \hat{\rho}(\hat{x}) \stackrel{\text { def }}{=} \frac{\rho(L \hat{x})}{\rho_{0}} \\
& \hat{\rho}^{*}(\hat{t}) \stackrel{\text { def }}{=} \frac{\rho^{*}\left(t_{\text {proc }} \hat{t}\right)}{\rho_{0}} \\
& \hat{\mathscr{A}}(\hat{x}) \stackrel{\text { def }}{=} \frac{\mathscr{A}(L \hat{x})}{\mathscr{A}_{0}}
\end{aligned}
$$


It should be noted that $\hat{\rho}^{*}(\hat{\theta})=\hat{\rho}(\hat{x})$ since density depends on the composition only, as a consequence of Equation (14). Using these definitions, Equations (9), (13) and (21) become:

$$
\begin{gathered}
\frac{d \hat{Q}}{d \hat{x}}=\hat{f} \\
\frac{d \hat{\theta}}{d \hat{x}}=\frac{\frac{L \rho_{0} \mathscr{A}_{0}}{t_{p r o c} Q_{0}} \hat{\rho}^{*}(\hat{\theta}) \hat{\mathscr{A}}(\hat{x})}{\hat{Q}}=\frac{K \hat{\rho}^{*}(\hat{\theta}) \hat{\mathscr{A}}(\hat{x})}{\hat{Q}} \\
\hat{f}_{i}=\frac{L \rho_{0} \mathscr{A}_{0}}{t_{\text {proc }} Q_{0}} \hat{\rho}^{*}(\hat{\theta}) \hat{\mathscr{A}}(\hat{x}) \frac{F_{i}(\theta) t_{\text {proc }}}{m(\theta)}=K \hat{\rho}^{*}(\hat{\theta}) \hat{\mathscr{A}}(\hat{x}) \frac{F_{i}(\theta) t_{\text {proc }}}{m(\theta)}
\end{gathered}
$$

where $\hat{Q}(0)=1, \hat{\theta}(0)=0$ and

$$
K \stackrel{\text { def }}{=} \frac{L \rho_{0} \mathscr{A}_{0}}{t_{\text {proc }} Q_{0}}=\frac{\frac{L}{\mathrm{v}_{0}}}{t_{\text {proc }}}=\frac{\tau_{0}}{t_{\text {proc }}}=\frac{L}{L_{0}}
$$

is a dimensionless number expressing the ratio of the initial residence time with respect to the overall process time, which can also be considered as a dimensionless tubular reactor length. Here $\mathrm{v}_{0}=\frac{Q_{0}}{\rho_{0} \mathscr{A}_{0}}$ is the initial velocity, $\tau_{0}=\frac{L}{\mathrm{v}_{0}}$ is a formal initial residence time (that is, the one computed without accounting for the side feed) and $L_{0}=\frac{\mathrm{v}_{0}}{t_{\text {proc }}}$ is the reactor length computed without considering the side injections.

By further defining

$$
\hat{F}_{i}(\hat{\theta}) \stackrel{\text { def }}{=} F_{i}\left(t_{\text {proc }} \hat{\theta}\right) \frac{t_{\text {proc }}}{m_{0}}
$$

one gets

$$
\begin{aligned}
\frac{F_{i}(\theta)}{m(\theta)} & =\frac{F_{i}(\theta)}{m_{0}+\int_{0}^{\theta} F(t) d t}= \\
& =\frac{1}{t_{\text {proc }}} \frac{\hat{F}_{i}(\hat{\theta})}{1+\int_{0}^{\hat{\theta}} \hat{F}(\hat{t}) d \hat{t}} \stackrel{\text { def }}{=} \frac{\hat{\gamma}_{i}(\hat{\theta})}{t_{\text {proc }}}
\end{aligned}
$$

and Equation (25) becomes:

$$
\frac{d \hat{Q}}{d \hat{x}}=\sum_{i=1}^{N C} \hat{\gamma}_{i}(\hat{\theta})
$$

By applying the chain rule and using Equation (26) it is possible to obtain:

$$
\frac{d \hat{Q}}{d \hat{\theta}}=K \hat{\rho}^{*}(\hat{\theta}) \hat{\mathscr{A}}(\hat{x}) \sum_{i=1}^{N C} \hat{\gamma}_{i}(\hat{\theta})\left(\frac{d \hat{\theta}}{d \hat{x}}\right)^{-1}=\hat{Q} \sum_{i=1}^{N C} \hat{\gamma}_{i}(\hat{\theta})
$$


This equation can be integrated, leading to

$$
\begin{aligned}
\hat{Q}(\hat{\theta}) & =\exp \left(\int_{0}^{\hat{\theta}} \sum_{i=1}^{N C} \hat{\gamma}_{i}(\hat{t}) d \hat{t}\right)= \\
& =1+\int_{0}^{\hat{\theta}} \hat{F}(\hat{t}) d \hat{t}
\end{aligned}
$$

where

$$
\hat{F}(\hat{t})=\sum_{i=1}^{N C} \hat{F}_{i}(\hat{t})
$$

which allows solving Equation (26) as:

$$
\int_{0}^{\hat{\theta}} \frac{\hat{Q}(\hat{t})}{\hat{\rho}^{*}(\hat{t})} d \hat{t}=K \int_{0}^{\hat{x}} \hat{\mathscr{A}}(\hat{\xi}) d \hat{\xi}
$$

to obtain the function $\hat{\theta}(\hat{x})$.

$K$ value can be found by using the dimensionless Equation (15)

$$
\hat{\theta}(1)=1
$$

together with Equation (35)

$$
K=\frac{\int_{0}^{1} \frac{\hat{Q}(\hat{\theta})}{\hat{\rho}^{*}(\hat{\theta})} d \hat{\theta}}{\int_{0}^{1} \hat{\mathscr{A}}(\hat{x}) d \hat{x}}
$$

Consequently, Equation (27) becomes:

$$
\hat{f}_{i}(\hat{x})=K \hat{\rho}^{*}(\hat{\theta}) \hat{\mathscr{A}}(\hat{x}) \frac{\hat{F}_{i}(\hat{\theta})}{\hat{Q}(\hat{\theta})}
$$

The solution of this problem is guaranteed to exist and to be unique by the Cauchy-Lipschitz theorem. In fact the problem consists in the solution of a Cauchy problem: the integrand function $\hat{\theta}(\hat{x})$ is Lipschitz continuous on $[0,1]$ as it has bounded first derivative, therefore satisfying the theorem conditions.

Note that the trivial case with constant density and cross sectional area leads to the simpler equation:

$$
K=\int_{0}^{1} \hat{Q}(\hat{\theta}) d \hat{\theta}
$$

The general algorithm used to solve this problem is the following: 
1. assign a value to $\mathscr{A}(\hat{x})$ and $Q_{0}$;

2. from the SBR recipe, compute $\hat{\rho}^{*}(\hat{t}), \hat{F}_{i}(\hat{t})$, and $\hat{F}(\hat{t})$ through Equations (24), (29) and (34);

3. compute $\hat{Q}(\hat{\theta})$ through Equation (33);

4. compute $K$ through Equation (37);

5. solve ODE (26) to compute $\hat{\theta}(\hat{x})$;

6. compute $\hat{f}_{i}(\hat{x})$ using Equation (38);

7. find the tubular reactor length using Equation (28).

From these dimensionless values, dimensional values can be easily computed through their definitions (24).

An important feature of the proposed procedure is now evident: no kinetic information is required once an effective recipe is available. In other words, some measurements on the industrial SBR (namely: $\rho^{*}(t), \underline{F}(t)$, and $m_{0}$ ) suffice to identify all the parameters of a side fed tubular reactor which is able to achieve the same product quality (at least in terms of conversion and selectivity) of an existing SBR industrial plant.

\section{Parametric analysis}

The proposed procedure guarantees a rigorous transformation of a SBR based process to a continuous one carried out in a Zwietering-like tubular reactor. However, also more trivial procedures, based on rulesof-thumb arising from engineering intuition, could be guessed. To highlight the different results (in terms of conversion and selectivity) obtained using the proposed methodology or a more intuitive side feed injection policy, a simple case study was investigated. Density and cross sectional area are assumed constant for the sake of simplicity and a second-order process reaction is considered:

$$
A+B \rightarrow C \quad r_{1}=k_{1} c_{A} c_{B}
$$

along with an undesired first-order side reaction consuming the desired product, $C$ :

$$
C \rightarrow D \quad r_{2}=k_{2} c_{C}
$$

According to the SBR recipe, a given amount of the main reactant $B, m_{0}$, has to be initially loaded in the reactor, while the coreactant $A$ is fed at constant flowrate, $F_{A 0}$. For this simple case study, an analytical solution can be found by applying the aforementioned procedure: 
1. arbitrary values can be chosen for both $\mathscr{A}_{0}$ and $Q_{0}$; however, being the cross sectional area constant, it follows that:

$$
\hat{\mathscr{A}}=1
$$

2. $\hat{\rho}^{*}(\hat{t}), \hat{F}_{i}(\hat{t})$ and $\hat{F}(\hat{t})$ are computed using Equations (24), (29) and (34), resulting in the following constant values:

$$
\begin{gathered}
\hat{\rho}^{*}=1 \\
\hat{F}=\hat{F}_{A}=\frac{t_{\text {proc }}}{m_{0}} F_{A 0} \quad \hat{F}_{B}=\hat{F}_{C}=\hat{F}_{D}=0
\end{gathered}
$$

3. $\hat{Q}(\hat{\theta})$ is computed through Equation (33), leading to:

$$
\hat{Q}(\hat{\theta})=1+\hat{F} \hat{\theta}
$$

4. $K$ is then computed using Equation (37):

$$
K=1+\frac{\hat{F}}{2}
$$

5. the ODE (26) can be integrated analytically to give the functional dependence $\hat{\theta}(\hat{x})$ :

$$
\hat{\theta}(\hat{x})=\frac{1}{\hat{F}}(\sqrt{1+2 K \hat{F} \hat{x}}-1)
$$

6. $\hat{f}_{A}(\hat{x})$ is computed using Equation (38) (note that only the coreactant $A$ is fed to the SBR and therefore injected laterally in the tubular reactor):

$$
\hat{f}_{A}(\hat{x})=\frac{K \hat{F}}{\sqrt{1+2 K \hat{F} \hat{x}}}
$$

7. finally, $\hat{f}_{A}(\hat{x})$ is made dimensional using Equation (38) and the tubular reactor length is found using Equation (28)

$$
\begin{aligned}
& f_{A}(x)=\frac{\rho_{0} \mathscr{A}_{0} F_{A 0}}{m_{0} \sqrt{1+2 \frac{\rho_{0} \mathscr{A}_{0} F_{A 0}}{m_{0} Q_{0}} x}} \\
& L=\left(1+\frac{F_{A 0} t_{\text {proc }}}{2 m_{0}}\right) \frac{Q_{0} t_{p r o c}}{\rho_{0} \mathscr{A}_{0}}
\end{aligned}
$$

As previously stated, it is important noting that no chemical kinetics information was used to evaluate $f_{A}(x)$ 
and $L$.

This solution can be compared with an approximate solution derived from a more intuitive transformation involving constant side feedrate, $f_{A 0}$, all along the entire tubular reactor. For instance, this constant side feedrate can be computed using the following similarity relationship between tubular reactor flowrates and SBR masses:

$$
\frac{f_{A 0} L}{Q_{0}}=\frac{F_{A 0} t_{\text {proc }}}{m_{0}}
$$

Moreover, the mean residence time in the tubular reactor can be set equal to the SBR process time as follows:

$$
\frac{\rho \mathscr{A} L}{Q_{0}+\frac{f_{A 0} L}{2}}=t_{\text {proc }}
$$

Also the use of these rules-of-thumb leads to analytical relations for both $f_{A 0}$ and $L$ :

$$
\begin{gathered}
f_{A 0}=\frac{\rho_{0} \mathscr{A}_{0} F_{A 0}}{m_{0}\left(1+\frac{F_{A 0} t_{\text {proc }}}{2 m_{0}}\right)} \\
L=\left(1+\frac{F_{A 0} t_{\text {proc }}}{2 m_{0}}\right) \frac{Q_{0} t_{\text {proc }}}{\rho_{0} \mathscr{A}_{0}}
\end{gathered}
$$

The two different lateral feed policies are compared in Figure 3 for different values of the parameter $\hat{F}$, which represents the amount of coreactant fed to the SBR with respect to the initially loaded amount of reactants. At increasing values of $\hat{F}$, the variation of the required lateral feed with the axial coordinate increases and, therefore, the differences between the two feed laws increase as well.

In particular, it is worthwhile noting that the constant feed policy of the SBR results in a non-constant lateral feed flowrate along the axial coordinate of the tubular reactor. This behaviour arises from the constraint (22): being $\mathscr{A}$ and $F_{A}$ constant in this case study, the time variation of the SBR reaction volume results in a $f_{A}$ change along the axial coordinate of the tubular reactor. Note that, in spite of the linear variation of the volume in time, the axial variation of $f_{A}$ is not linear due to the non-linear dependence of $\theta$ on $x$.

The practical impact of the different feed policies can be estimated by integrating the mass balances (Equations (7) and (9)) for both constant and variable lateral feed policies. Then, the outlet flowrates of the desired product $C$ computed using the process parameters summarised in Tables 1 and 2 are compared through their percentage relative difference, defined as:

$$
\Delta Q_{C}=\left|\frac{Q_{C}-Q_{C}^{c o n s t}}{Q_{C}}\right|_{x=L} \cdot 100
$$




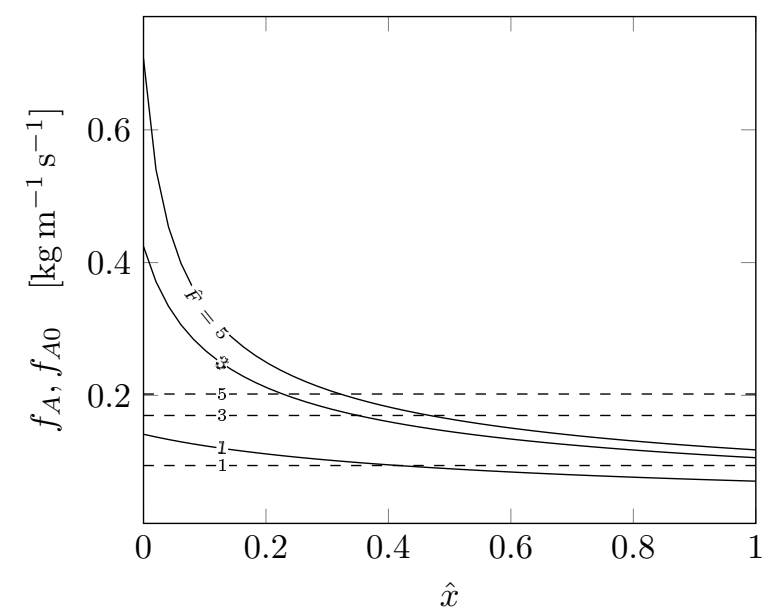

Figure 3: Side feed flowrate along the dimensionless axial coordinate for the rigorous (solid line) and for the constant lateral feed (dashed line) transformations. Curves are parametrised according to the dimensionless SBR feedrate, $\hat{F}$.

Table 1: Process data for parametric analysis simulations.

\begin{tabular}{ll}
\hline$M W_{A}=M W_{B}$ & $20 \mathrm{~kg} \mathrm{kmol}^{-1}$ \\
$M W_{C}=M W_{D}$ & $40 \mathrm{~kg} \mathrm{kmol}^{-1}$ \\
$m_{0}$ & $1000 \mathrm{~kg}$ \\
$t_{\text {proc }}$ & $500 \mathrm{~s}$ \\
$\rho$ & $1000 \mathrm{~kg} \mathrm{~m}^{-3}$ \\
$Q_{0}$ & $1 \mathrm{~kg} \mathrm{~s}^{-1}$ \\
$\mathscr{A}$ & $0.07 \mathrm{~m}^{2}$ \\
\hline
\end{tabular}

in Figure 4 as a function of the dimensionless SBR feed rate, $\hat{F}$, parametrised according to the kinetic constant values of the two reactions, $k_{1}$ and $k_{2} . Q_{C}$ is the flowrate computed using the rigorous transformation policy, while $Q_{C}^{\text {const }}$ is that computed using constant lateral feed flowrate. We can see that large differences arise, clearly indicating that the proposed approach is more effective than simpler rules-of-thumb in the general case.

Another comparison was performed between the continuous lateral feed injection and a discrete one, to investigate the possibility of implementing the proposed procedure with a series of finite additions. This was done by dividing the computed reactor length in $N_{d}$ equal parts, each having a lateral feed injection at

Table 2: Reaction rate constants for the selected cases.

\begin{tabular}{ccr}
\hline & $k_{1} \cdot 10^{6}$ & $k_{2} \cdot 10^{4}$ \\
& {$\left[\frac{\mathrm{m}^{3}}{\mathrm{kmol} \mathrm{s}}\right]$} & {$\left[\mathrm{s}^{-1}\right]$} \\
$\mathrm{a}$ & 1 & 1 \\
$\mathrm{~b}$ & 1 & 100 \\
$\mathrm{c}$ & 100 & 1 \\
$\mathrm{~d}$ & 100 & 100 \\
\hline
\end{tabular}




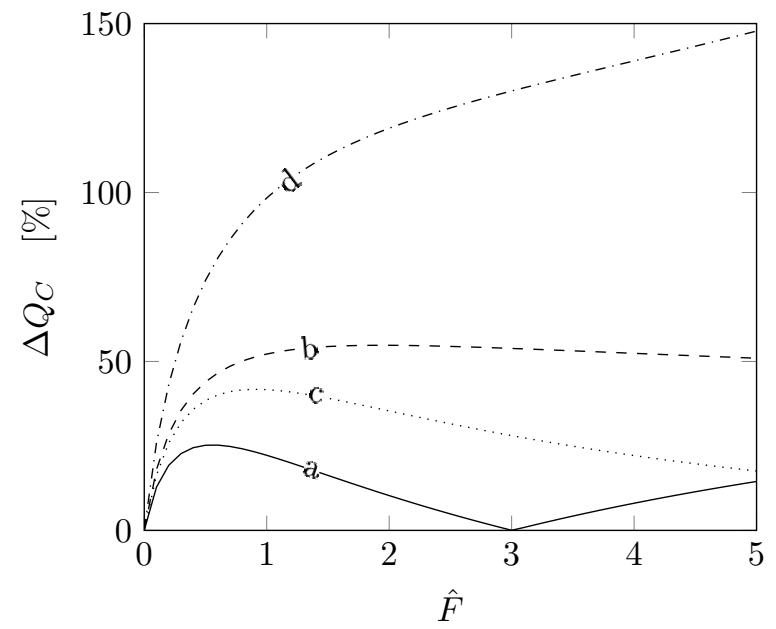

Figure 4: Percentage relative difference between outlet flowrates of species $C$ (Equation (55)) computed using the constant side feed policy and the rigorous one. Curves are parametrised according to the kinetic constants, as from Table 2.

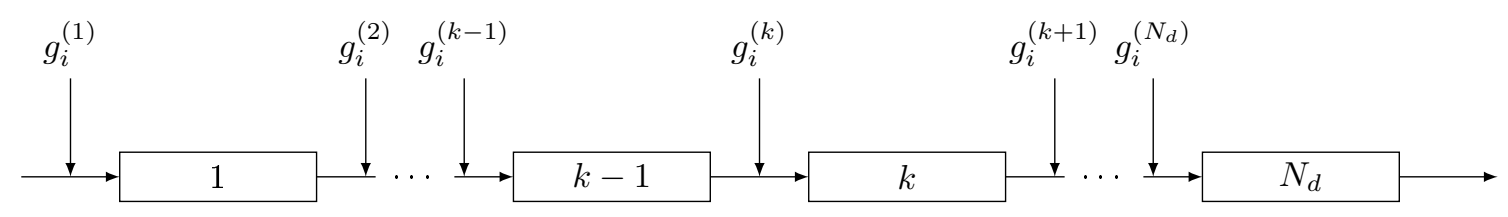

Figure 5: Approximation of the continuous lateral feed injection, $f_{i}(x)$, with $N_{d}$ discrete side injections, $g_{i}^{(k)}$.

its beginning, with a mass flowrate equal to

$$
g_{i}^{(k)}=\int_{\frac{L}{N_{d}}(k-1)}^{\frac{L}{N_{d}} k} f_{i}(x) d x \quad k=1, \ldots, N_{d}
$$

as shown in Figure 5; obviously, $L$ and $f_{i}(x)$ values are computed using the rigorous approach.

Modelling such reactor configuration requires the solution of $N_{d} \cdot N C$ PFR mass balance equations:

$$
\frac{d Q_{i}^{(k)}}{d x}=\dot{\Omega}_{i}\left(\underline{\omega}^{(k)}\right)
$$

where $Q_{i}^{k}$ is the mass flowrate of species $i$ along the axial coordinate of the $k$-th section, and

$$
\omega_{i}^{(k)}=\frac{Q_{i}^{(k)}}{\sum_{j=1}^{N C} Q_{j}^{(k)}}
$$

These equations are solved with BCs:

$$
Q_{i}^{(k)}(0)=Q_{i, \text { out }}^{(k-1)}+g_{i}^{(k)}
$$




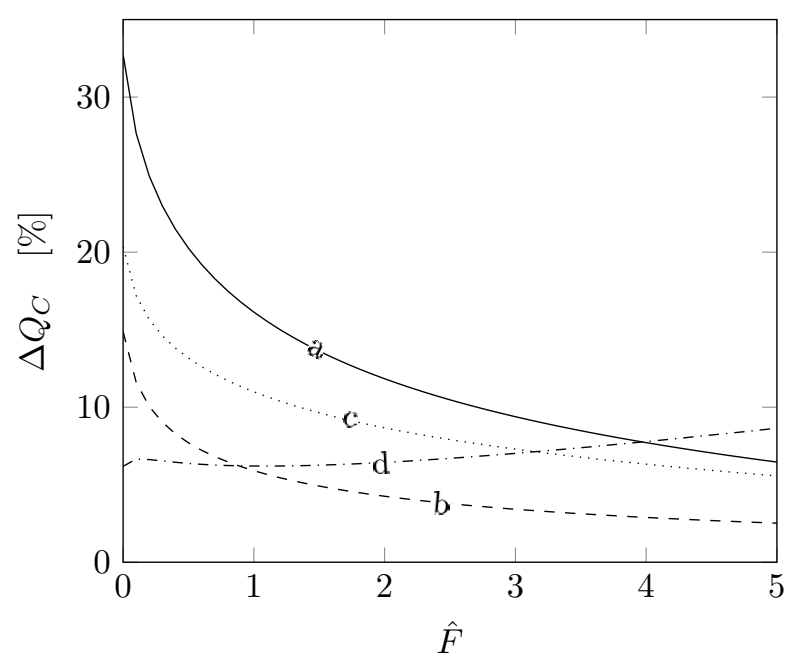

(a) $N_{d}=3$

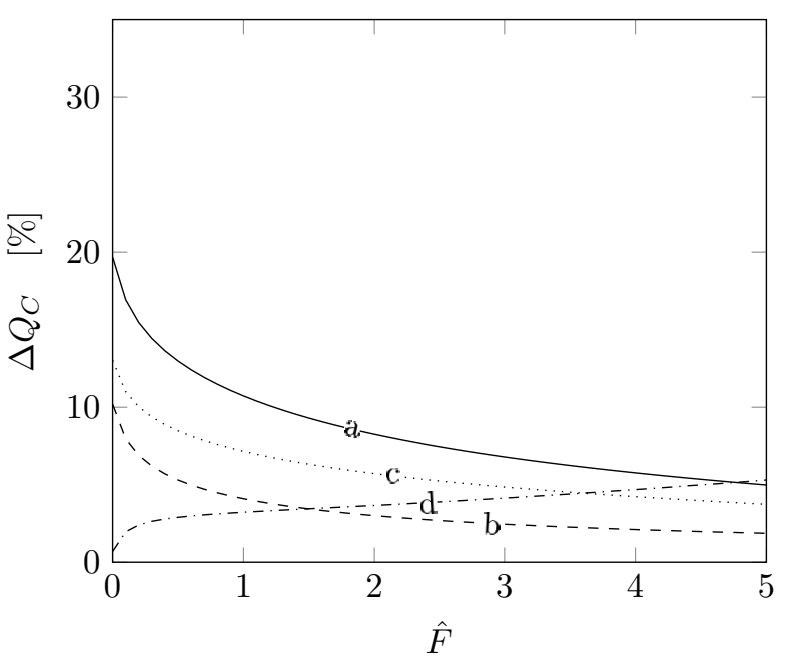

(b) $N_{d}=5$

Figure 6: Percentage relative difference between outlet flowrates for species $C$ (Equation (55)) using the discrete side feed policy with respect to the rigorous one. Curves are parametrised according to the kinetic constants, as from Table 2 .

where $Q_{i, \text { out }}^{(k-1)}$ is the flowrate of species $i$ at the outlet of the previous $(k-1)$ section, defined as:

$$
Q_{i, \text { out }}^{(k-1)}= \begin{cases}Q_{0} \omega_{i, 0} & k=1 \\ \left.Q_{i}^{(k-1)}\right|_{x=\frac{L}{N_{d}}} & k>1\end{cases}
$$

Figure 6 compares the continuous and discrete feed policies for different values of the kinetic constants in terms of $\Delta Q_{C}$ as defined by Equation (55). Even though the differences are not negligible, they are definitely lower than those found using a constant side feed rate policy and obviously decrease by increasing the number of subdivisions, as can be seen from Figures 7 and 8 . The former shows how the maximum difference in productivity changes according to the number of discrete feeds, for any analysed dimensionless feedrate and couple of kinetic constants. The latter depicts $C$ mass fractions according to the number of feed locations for an example case. Differences decrease super-linearly between the ideal continuous feed policy and the discrete one at increasing number of feed points. If a maximum deviation of $5 \%$ can be accepted, a critical value (above which deviations are smaller) of $N_{d}=20$ is obtained. 


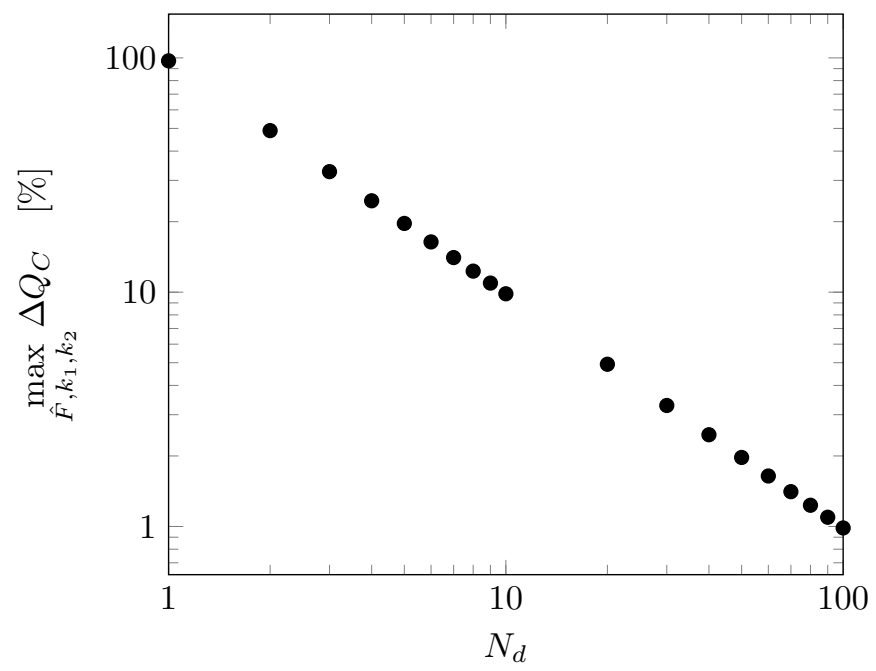

Figure 7: Maximum relative difference between outlet flowrates for species $C$ using the discrete side feed policy with respect to the rigorous one as function of the number of discrete feeds.

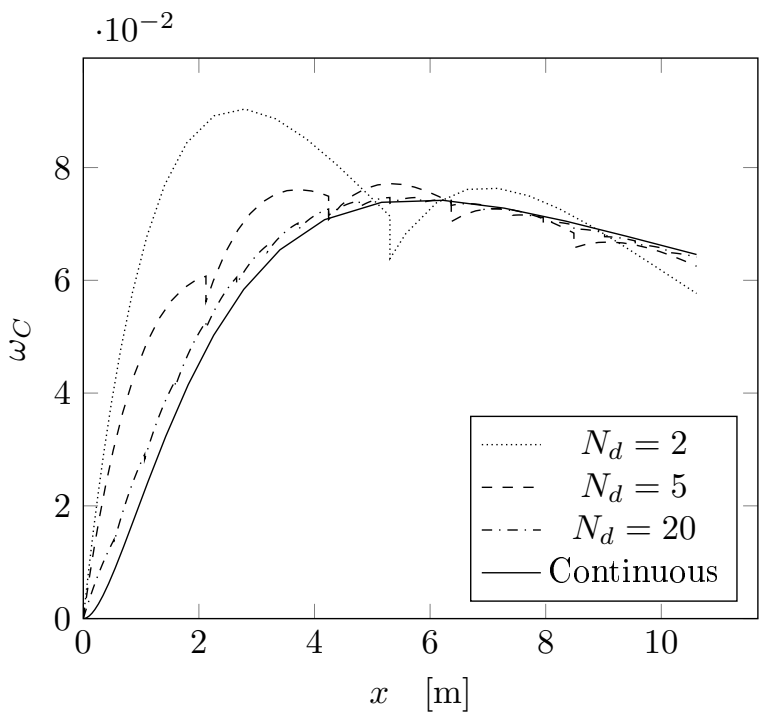

Figure 8: $C$ mass fraction along the axial coordinate where $\hat{F}=1, \quad k_{1}=1 \times 10^{-4} \mathrm{~m}^{3} \mathrm{kmol}^{-1} \mathrm{~s}^{-1}$ and $k_{2}=1 \times 10^{-2} \mathrm{~m}^{3} \mathrm{kmol}^{-1} \mathrm{~s}^{-1}$. Curves are parametrised according to the number of discrete feeds. 


\section{Case studies}

The potentials of the proposed approach were investigated through two case studies, representative of two relevant classes of industrial processes: production of copolymers and production of fine chemicals.

\subsection{Copolymerisation}

\subsubsection{SBR feed policy design}

The feedrate of a copolymerisation SBR can be designed to obtain a given product composition by means of the well known power feed policy $[15,16]$. According to this feed policy, the rate between the residual amounts of the two monomers ( $A$, the most reactive, and $B$, the least reactive) is kept constant during the entire process, thus enabling the production of a copolymer at constant composition, i.e. with the desired monomer $i$ mass fraction, $\phi_{i}^{w}$. A typical reaction scheme for a radical copolymerisation process involves initiation, propagation and termination reactions, as summarised in Table 3. According to the power feed policy, $A$ is the coreactant to be fed to the SBR, while the stoichiometric amount of $B$ is charged to the reactor at the beginning of the process. Therefore, molar balances for the two monomers in the SBR are:

$$
\left\{\begin{array}{l}
\frac{d n_{A}}{d t}=\dot{N}_{A}(t)+R_{A} V \\
\frac{d n_{B}}{d t}=R_{B} V
\end{array}\right.
$$

where $n_{i}(t)$ is the molar quantity of species $i, \dot{N}_{A}$ is the feedrate of $A$ to the SBR, $R_{i}=-k_{p i}^{*} c_{R} \bullet c_{i}$ is species $i$ molar production rate, $c_{i}$ is species $i$ molar concentration and $c_{R}$. is the overall concentration of active chains, given by the quasi-steady-state formula $\sqrt{\frac{R_{I}}{k_{t}^{*}}}$. Assuming long chains approximation (LCA) [17] and the pseudo-kinetic approach [18], the expressions of effective rate coefficients (indicated by the superscript $*$ ) can be readily evaluated as a function of the actual monomer mixture composition as summarised in Table 4.

The analytical solution of these equations is:

$$
\begin{gathered}
n_{B}(t)=\frac{n_{B, 0}}{n_{A, 0}} n_{A}(t)=n_{B, 0} e^{-\tilde{k}_{d} t} \\
\dot{N}_{A}(t)=\dot{N}_{A, 0} e^{-\tilde{k}_{d} t}
\end{gathered}
$$

where $n_{A, 0}$ and $n_{B, 0}$ can be obtained from the so called Mayo-Lewis plot [19], which reports monomer $A$ molar fraction of the instantaneously produced copolymer, $\phi_{A}$, as a function of the same monomer molar 
Table 3: Radicalic copolymerisation reaction scheme. All reactions are bimolecular second order, except initiation, which is unimolecular with rate $R_{I}$. $A_{l, m}^{\bullet}$ stands for a chain of $l$ units of $A$ and $m$ units of $B$, where the radical is located on the terminating $A$ unit.

\begin{tabular}{lll}
\hline & Reaction & Rate constant \\
\hline Initiation & $I_{2}+M \rightarrow 2 R^{\bullet}$ & \\
Propagation & $A_{l, m}^{\bullet}+A \rightarrow A_{l+1, m}^{\bullet}$ & $k_{p A A}$ \\
& $A_{l, m}^{\bullet}+B \rightarrow B_{l, m+1}^{\bullet}$ & $k_{p A B}$ \\
& $B_{l, m}^{\bullet}+A \rightarrow A_{l+1, m}^{\bullet}$ & $k_{p B A}$ \\
& $B_{l, m}^{\bullet}+B \rightarrow B_{l, m+1}^{\bullet}$ & $k_{p B B}$ \\
Termination & $A_{l, m}^{\bullet}+A_{q, r}^{\bullet} \rightarrow P_{l+q, m+r}$ or $P_{l, m}+P_{q, r}$ & $k_{t A A}$ \\
& $A_{l, m}^{\bullet}+B_{q, r}^{\bullet} \rightarrow P_{l+q, m+r}$ or $P_{l, m}+P_{q, r}$ & $k_{t A B}$ \\
& $B_{l, m}^{\bullet}+B_{q, r}^{\bullet} \rightarrow P_{l+q, m+r}$ or $P_{l, m}+P_{q, r}$ & $k_{t B B}$ \\
\hline
\end{tabular}

Table 4: Variable definitions for the copolymerisation case study.

\begin{tabular}{|c|c|}
\hline Variable & Definition \\
\hline$R_{I}$ & $2 \eta k_{d} c_{I_{2}}$ \\
\hline$k_{p A}^{*}$ & $k_{p A A} p_{A}+k_{p B A} p_{B}$ \\
\hline$k_{p B}^{*}$ & $k_{p B B} p_{B}+k_{p A B} p_{A}$ \\
\hline$p_{A}$ & $\frac{k_{p B A} c_{A}}{k_{p A B} c_{B}+k_{p B A} c_{A}}$ \\
\hline$p_{B}$ & $\frac{k_{p A B} c_{B}}{k_{p A B} c_{B}+k_{p B A} c_{A}}$ \\
\hline$k_{t}^{*}$ & $k_{t A A} p_{A}^{2}+2 k_{t A B} p_{A} p_{B}+k_{t B B} p_{B}^{2}$ \\
\hline$\tilde{k}_{d}$ & $\left(k_{p A B} k_{p B A} \frac{n_{A, 0}}{n_{B, 0}}+k_{p B B} k_{p A B}\right) \sqrt{\frac{R_{I}}{k_{t A A} k_{p B A}^{2}\left(\frac{n_{A, 0}}{n_{R} 0}\right)^{2}+2 k_{t A B} k_{p A B} k_{p B A} \frac{n_{A, 0}}{n_{B} 0}+k_{t B B} k_{p A B}^{2}}}$ \\
\hline$\dot{N}_{A, 0}$ & $n_{A 0}\left(k_{p A A} k_{p B A} \frac{n_{A 0}}{n_{B 0}}+k_{p A B} k_{p B A}\left(1-\frac{n_{A 0}}{n_{B 0}}\right)-k_{p B B} k_{p A B}\right)$. \\
\hline & $\cdot \sqrt{\frac{R_{I}}{k_{t A A} k_{p B A}^{2}\left(\frac{n_{A, 0}}{n_{B, 0}}\right)^{2}+2 k_{t A B} k_{p A B} k_{p B A} \frac{n_{A, 0}}{n_{B, 0}}+k_{t B B} k_{p A B}^{2}}}$ \\
\hline
\end{tabular}

fraction in the monomeric mixture, $X_{A}$ :

$$
\begin{aligned}
\phi_{A} & =\frac{\left(r_{A}-1\right) X_{A}^{2}+X_{A}}{\left(r_{A}-2\right) X_{A}^{2}+2 X_{A}+r_{B}\left(1-X_{A}\right)^{2}} \\
X_{A} & =\frac{n_{A}}{n_{A}+n_{B}}
\end{aligned}
$$

under the long-chain approximation. In these equations $r_{i}=\frac{k_{p i i}}{k_{p i j}}$ is the reactivity ratio of species $i$. From the $\phi_{A}$ value, using the known values of the monomer molecular weights, one can easily compute the moles of $A$ and $B$ required to produce a given mass of copolymer, $n_{A}^{\text {tot }}$ and $n_{B}^{\text {tot }}$. Since all the amount of $B$ is initially loaded into the SBR, from the $X_{A}$ value also the moles of $A$ to be initially loaded, $n_{A, 0}$, can be easily computed. The moles of $A$ to be fed to the SBR, $n_{A}^{\text {tot }}-n_{A, 0}$, can be related to the feed flowrate as 
Table 5: Process data for copolymerisation reaction.

\begin{tabular}{llc}
\hline & $A$ & \multicolumn{1}{c}{$B$} \\
\hline$M W_{i}\left[\mathrm{~kg} \mathrm{kmol}^{-1}\right]$ & 50 & \multicolumn{1}{c}{80} \\
$\rho\left[\mathrm{kg} \mathrm{m}^{-3}\right]$ & \multicolumn{2}{c}{900} \\
$F_{i}^{w}$ & 0.4 & 0.6 \\
$k_{p i i}\left[\mathrm{~m}^{3} \mathrm{kmol}^{-1} \mathrm{~s}^{-1}\right]$ & $3 \times 10^{3}$ & $1 \times 10^{3}$ \\
$r_{i}$ & 3 & 0.83 \\
$k_{t}\left[\mathrm{~m}^{3} \mathrm{kmol}^{-1} \mathrm{~s}^{-1}\right]$ & \multicolumn{2}{c}{$1 \times 10^{6}$} \\
$\eta k_{d}\left[\mathrm{~s}^{-1}\right]$ & \multicolumn{2}{c}{$1 \times 10^{-4}$} \\
\hline
\end{tabular}

follows:

$$
n_{A}^{t o t}-n_{A, 0}=\int_{0}^{t_{p r o c}} \dot{N}_{A}(t) d t=\int_{0}^{t_{p r o c}} \dot{N}_{A, 0} e^{-\tilde{k}_{d} t} d t
$$

This equation allows computing the overall process time:

$$
t_{\text {proc }}=-\frac{1}{\tilde{k}_{d}} \ln \left(1-\left(n_{A}^{\text {tot }}-n_{A, 0}\right) \frac{\tilde{k}_{d}}{\dot{N}_{A 0}}\right)
$$

This case study was worked out using the process data reported in Table 5 for a SBR aimed at producing $1 \mathrm{t}$ of copolymer. The initiator has initial concentration of $0.5 \mathrm{~mol} \%$.

Since the required value of $\phi_{A}$ is equal to 0.516 , the molar fraction of monomer $A$ in the reacting mixture, $X_{A}$, has to be equal to 0.357 from which the initial monomer amounts are readily evaluated: $n_{A 0}=4.167 \mathrm{kmol}, n_{B 0}=7.500 \mathrm{kmol}$, and $m_{0}=808.33 \mathrm{~kg}$. Therefore, using the parameter values in Table 4 , $\tilde{k}_{d}=3.604 \times 10^{-3} \mathrm{~s}^{-1}$ and $\dot{N}_{A 0}=13.82 \mathrm{~mol} \mathrm{~s}^{-1}$ are evaluated, leading to $t_{\text {proc }}=5200 \mathrm{~s}$. This process time is quite large because it reflects the continuously decreasing polymerisation rate when very high conversion is required. On the other hand, the required addition rate becomes negligible much before and the process can be interrupted much earlier without affecting product amount and quality. About $99 \%$ of the overall amount of monomer $A$ has been already injected into the reactor after slightly more than $1000 \mathrm{~s}$. This specific value has been set as the process duration, i.e. $t_{\text {proc }}=1074 \mathrm{~s}$.

\subsubsection{Transformation to continuous process}

For the sake of example, the tubular reactor is assumed to have an inlet total flowrate equal to $1 \mathrm{~kg} \mathrm{~s}^{-1}$ and a diameter of $0.3 \mathrm{~m}$. Assuming again constant density and cross sectional area, the transformation to a side-fed tubular reactor carried out using the previously discussed procedure leads to an analytical solution, summarised in the following:

1. assuming constant cross sectional area, $\hat{\mathscr{A}}=1$ follows; 
2. $\hat{\rho}^{*}(\hat{t}), \hat{F}_{i}(\hat{t})$ and $\hat{F}(\hat{t})$ are computed using Equations (24), (29) and (34), leading to:

$$
\begin{gathered}
\hat{\rho}=1 \\
\hat{F}(\hat{t})=\hat{F}_{A}(\hat{t})=a e^{-b \hat{t}} \quad \hat{F}_{B}=\hat{F}_{C}=\hat{F}_{D}=0
\end{gathered}
$$

where $a \stackrel{\text { def }}{=} \frac{\dot{N}_{A, 0} M W_{A} t_{\text {proc }}}{m_{0}}=0.918$ and $b \stackrel{\text { def }}{=} \tilde{k}_{d} t_{\text {proc }}=3.869$.

3. $\hat{Q}(\hat{\theta})$ is found from Equation (33):

$$
\hat{Q}(\hat{\theta})=1+\frac{a}{b}\left(1-e^{-b \hat{\theta}}\right)
$$

4. $K$ is computed from Equation (37):

$$
K=1+\frac{a}{b}+\frac{a}{b^{2}}\left(e^{-b}-1\right)=1.177
$$

5. the ODE (26) is solved to give a relationship between $\hat{\theta}$ and $\hat{x}$ :

$$
K \hat{x}=\left(1+\frac{a}{b}\right) \hat{\theta}+\frac{a}{b^{2}}\left(e^{-b \hat{\theta}}-1\right)
$$

6. $\hat{f}_{A}(\hat{\theta})$ is computed using Equation (38):

$$
\hat{f}_{A}(\hat{\theta})=\frac{K a b e^{-b \hat{\theta}}}{b+a\left(1-e^{-b \hat{\theta}}\right)}
$$

7. the tubular reactor length is computed using Equation (28):

$$
L=\frac{K t_{p r o c} Q_{0}}{\rho_{0} \mathscr{A}_{0}}=19.9 \mathrm{~m}
$$

8. $f_{A}(x)$ is computed using Equation (38) leading to the side feed profile shown in Figure 9.

From the numerical simulation of the side-fed tubular reactor using Equations (7) and (9), it can be easily verified that a constant value of $X_{A}=0.357$ is maintained throughout the entire reactor length: this means that the produced polymer will have the desired constant value of monomer ratio as in the SBR process.

Finally, it is worth mentioning again that kinetic information (i.e., $\dot{N}_{A 0}$ and $\tilde{k}_{d}$ ) is used only to derive the 


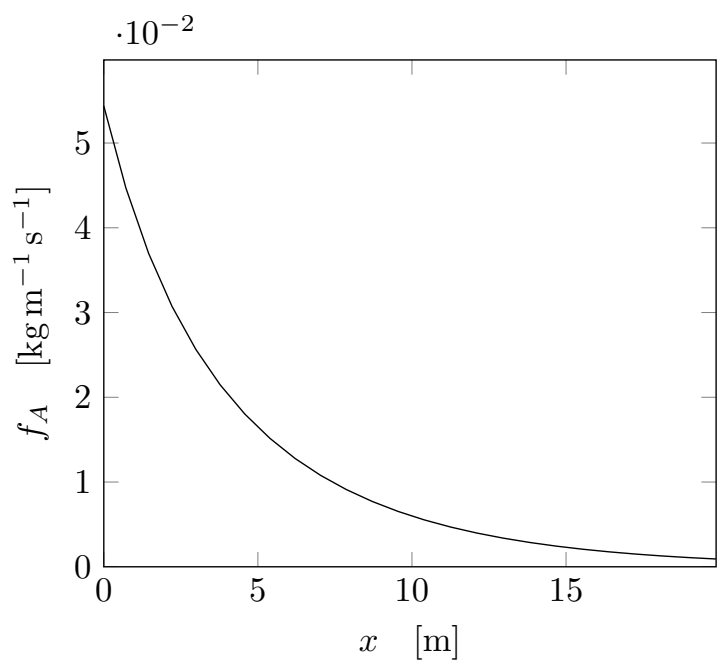

Figure 9: Side feed policy as a function of the axial coordinate for the copolymerisation reaction.

SBR feed policy. However, given the SBR feed policy (that is, given the value of $F_{i}(t)$ ), the transformation of the industrial SBR process into a tubular reactor with side injections can be carried out without any kinetic information.

\subsection{Fine chemicals}

This case study is inspired to the production of a diol, $D$, via catalytic reduction (catalyst, $C$ ) and hydrolysis (water, $W$ ) of the corresponding internal ester, $E$, as in the last step of the regioselective reaction discussed by Bringmann et al. [20]:

$$
\begin{aligned}
E+C & \rightarrow E C \\
r_{1} & =3 \times 10^{8} \exp \left(-\frac{7800}{T}\right) c_{E} c_{C} \\
E C+W & \rightarrow D+C \\
r_{2} & =1 \times 10^{8} \exp \left(-\frac{6800}{T}\right) c_{E C} c_{W}
\end{aligned}
$$

with $r\left[\mathrm{kmol} \mathrm{m}^{-3} \mathrm{~s}^{-1}\right], T[\mathrm{~K}]$ and $c_{i}\left[\mathrm{kmol} \mathrm{m}^{-3}\right] . E C$ is an intermediate complex and the reaction is carried out in a solvent, $S$.

The recipe developed at laboratory scale for the production of $D$ in a SBR is summarised in Table 6 . The reactor is initially charged with $10 \mathrm{~g} \mathrm{~S}$. Temperature is kept constant at $30^{\circ} \mathrm{C}$ throughout the entire process. 
Table 6: Laboratory scale recipe for the asymmetric synthesis.

\begin{tabular}{cl}
\hline Time $[\mathrm{s}]$ & Description \\
\hline $0-3600$ & Constant simultaneous feeding of $19.8 \mathrm{~g} 9 \mathrm{wt} \%$ solution \\
& of $E$ in $S$ and $18.36 \mathrm{~g} 2 \mathrm{wt} \%$ solution of $C$ in $S$. \\
$3600-3660$ & Idle. \\
$3660-3720$ & Constant feeding of $0.60 \mathrm{~g} \mathrm{W.}$ \\
$3720-4020$ & Idle. \\
\hline
\end{tabular}

Table 7: Process data for the diol synthesis.

\begin{tabular}{ll}
\hline$M W_{E}$ & $260 \mathrm{~kg} \mathrm{kmol}^{-1}$ \\
$M W_{C}$ & $80 \mathrm{~kg} \mathrm{kmol}^{-1}$ \\
$M W_{W}$ & $18 \mathrm{~kg} \mathrm{kmol}^{-1}$ \\
$\rho$ & $911 \mathrm{~kg} \mathrm{~m}^{-3}$ \\
$Q_{0}$ & $0.3 \mathrm{~kg} \mathrm{~s}^{-1}$ \\
$\mathscr{A}$ & $0.0314 \mathrm{~m}^{2}$ \\
\hline
\end{tabular}

Using the process data summarised in Table 7, the transformation of this SBR recipe to the one suitable for a single side-fed tubular reactor was performed through the previously discussed procedure, which in this case does not allow an analytical solution. The reactor length results equal to about $130 \mathrm{~m}$ with the feed policy summarised in Figure 10 for each species to be fed. Once more, to highlight the relevance of the use of variable feed flowrates, mass balance equations (7) and (9) were solved for both the rigorous solution and a simplified case, which assumes constant side feed flowrates. The latter were obtained through the intuitive relationships previously discussed in section 3. Since the SBR process involves four steps, the constant flowrates are computed for each tubular reactor region separately using the following relations:

$$
\begin{gathered}
\frac{f^{(k)} L^{(k)}}{Q_{0}^{(k)}}=\frac{F^{(k)} t^{(k)}}{m_{0}^{(k)}} \\
\frac{\rho \mathscr{A} L^{(k)}}{Q_{0}^{(k)}+\frac{f^{(k)} L^{(k)}}{2}}=t^{(k)}
\end{gathered}
$$

where $k=1, \ldots, 4$ specifies the considered SBR step or reactor portion. In this case, the total reactor length is the sum of the lengths of the four regions, which is equal to about $125 \mathrm{~m}$, and the side feed flowrate values for each chemical species are easily computed from the relations:

$$
\frac{f_{i}^{(k)}}{f^{(k)}}=\frac{F_{i}^{(k)}}{F^{(k)}}
$$

The results of this transformation are summarised in Figure 10 as dashed lines. In spite of the similarity 

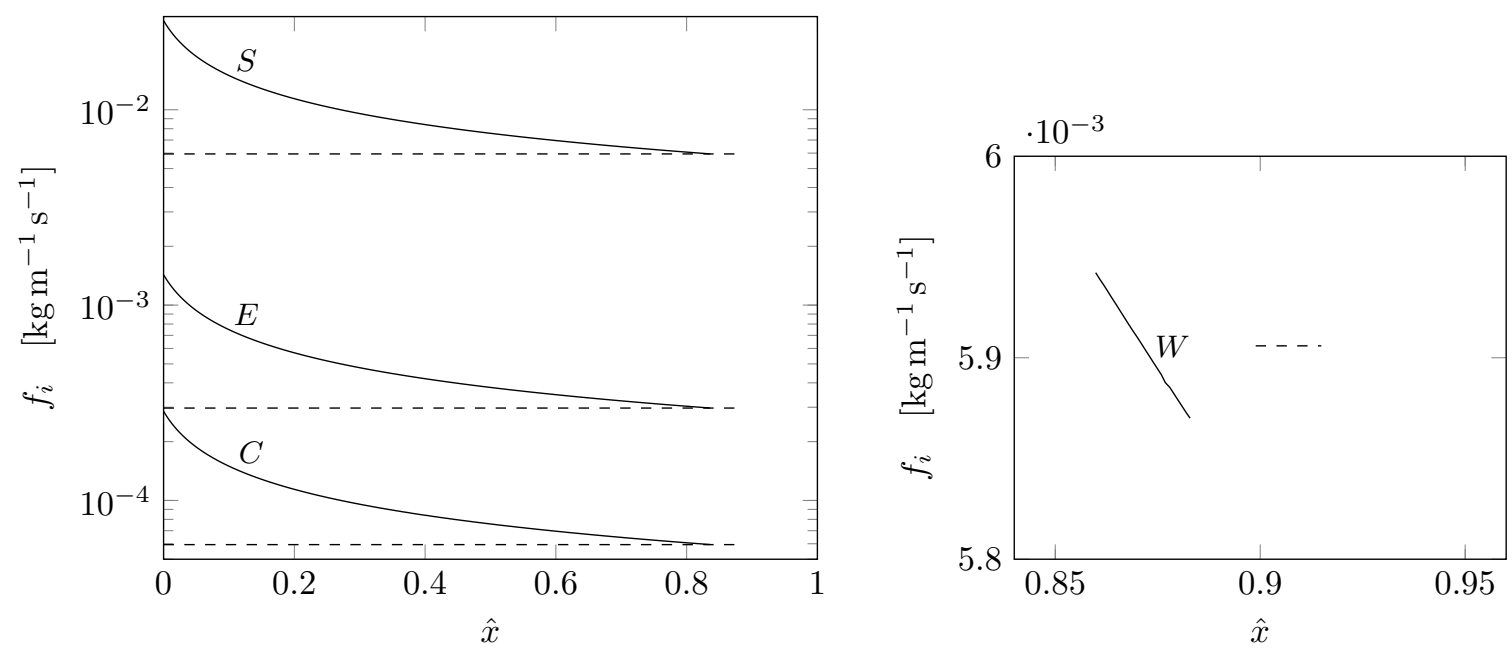

Figure 10: Side feed flowrate along the dimensionless axial coordinate according to the rigorous (solid line) and the constant feed (dashed line) method for the diol synthesis. Species $S, E$ and $C$ on the left, $W$ on the right (enlargement).

between these two side feed policies, they lead to quite different results in terms of tubular reactor performances: at constant side feed flowrates, about $40 \%$ less productivity is established than implementing the rigorous side feed flowrates. Furthermore, the final conversion is also reduced, by about $5 \%$. This means that the product quality from the constant feed tubular reactor is no more guaranteed to be the same as in the SBR plant. These results agree with the conclusions of the parametric analysis: since the rigorous solution is the only one that guarantees overlapping SBR and tubular reactor species profiles, the two reactor configurations show the same performances only in this case.

\section{Conclusions}

An approach for the transformation of isothermal homogeneous semi-batch processes into continuous ones was proposed and verified in the case of tubular reactors with distributed side injections. The key advantage of the proposed approach is that it does not require any kinetic information once the SBR recipe and some density measurements on an existing industrial plant are available. This procedure is optimal since it allows the exact reproduction of the original SBR performances. Of course, when available, kinetic information allow optimizing the reaction recipe whatever is the reactor type. However, since our primary focus is the transfer of a previously assessed recipe from SBR to the continuous tubular reactor, giving up the kinetic information becomes a major benefit.

Two case studies were analysed, a copolymer synthesis and the production of a fine chemical. In both cases, the tubular reactor ensures the same product composition and final conversion of the semi-batch 
process. Moreover, the reaction performances when applying these side feed policies have been compared with those achieved when using simpler and more intuitive feed policies, showing relevant differences.

Finally, it is worth mentioning that this tubular reactor enables scaling of productivity either changing the inlet flowrate or the cross sectional area. Being these two variables related to each other by well known design constraints, they act as a degree of freedom for the process design.

\section{Acknowledgements}

Financial support of Innovhub for one of us (FF) is gratefully acknowledged.

\section{Nomenclature}

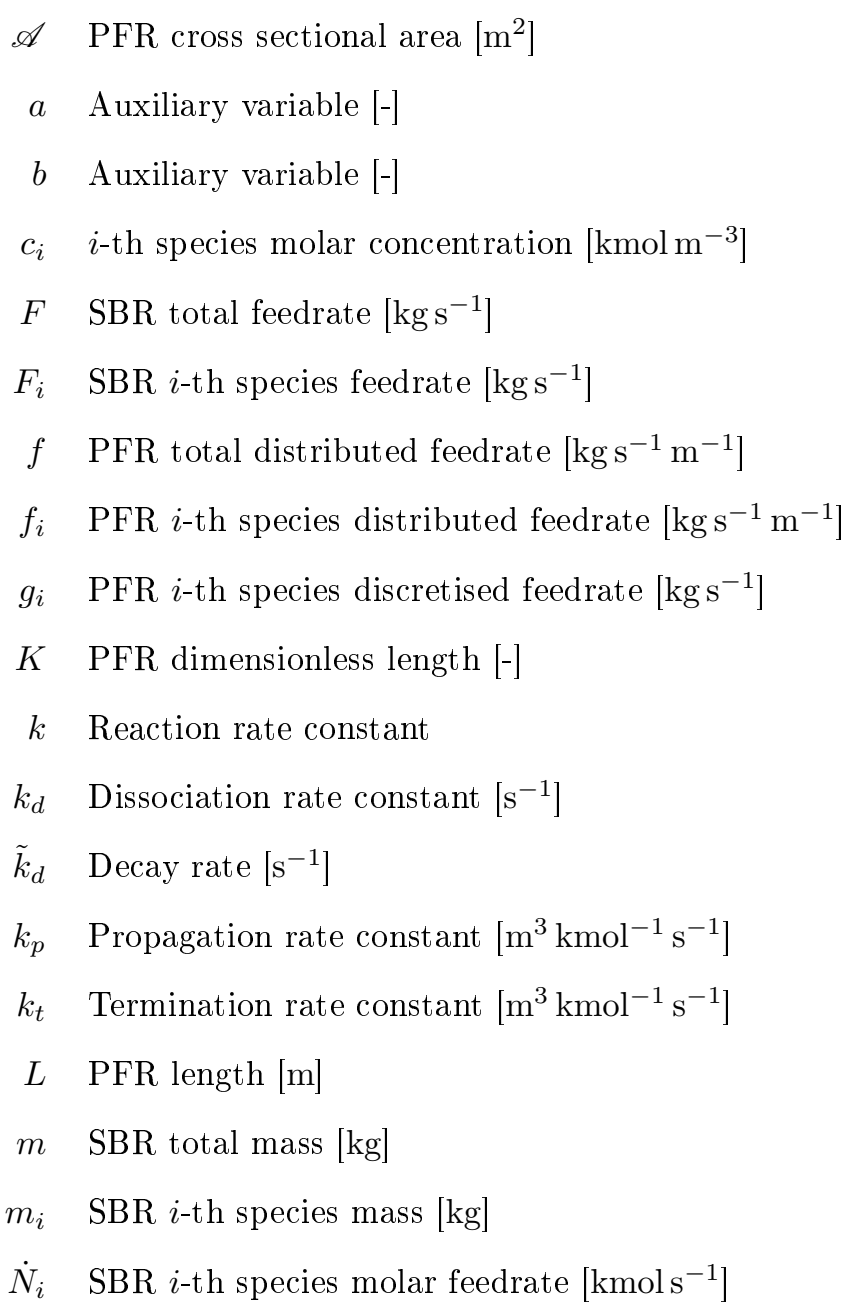


$n_{i} \quad$ SBR $i$-th species molar quantity [kmol]

$M W_{i} \quad i$-th species molecular weight $\left[\mathrm{kg} \mathrm{kmol}^{-1}\right]$

$N C \quad$ Number of components (species) [-]

$Q \quad$ PFR total flowrate $\left[\mathrm{kg} \mathrm{s}^{-1}\right]$

$Q_{i} \quad$ PFR $i$-th species flowrate $\left[\mathrm{kg} \mathrm{s}^{-1}\right]$

$R_{i} \quad i$-th species molar production rate $\left[\mathrm{kmol} \mathrm{m}^{-3} \mathrm{~s}^{-1}\right]$

$r \quad$ Molar reaction rate $\left[\mathrm{kmol} \mathrm{m}^{-3} \mathrm{~s}^{-1}\right]$

$r_{i} \quad i$-th species reactivity ratio [-]

$T \quad$ Temperature $[\mathrm{K}]$

$t \quad$ Time $[\mathrm{s}]$

$t_{\text {proc }}$ Overall process time [s]

$V \quad$ SBR volume $\left[\mathrm{m}^{3}\right]$

v Velocity $\left[\mathrm{m} \mathrm{s}^{-1}\right]$

$W_{i} \quad i$-th species overall mass $[\mathrm{kg}]$

$X_{i} \quad i$-th species monomeric molar fraction [-]

$x \quad$ PFR axial coordinate $[\mathrm{m}]$

\section{Greek symbols}

$\delta \quad$ Monomer fraction to be fed [-]

$\hat{\gamma}_{i} \quad$ Auxiliary variable [-]

$\phi_{i} \quad i$-th species desired molar fraction in copolymer [-]

$\phi_{i}^{w} \quad i$-th species desired mass fraction in copolymer [-]

$\dot{\Omega}_{i} \quad i$-th species massive production rate $\left[\mathrm{kg} \mathrm{m}^{-3} \mathrm{~s}^{-1}\right]$

$\omega_{i} \quad$ PFR $i$-th species mass fraction [-]

$\omega_{i}^{*} \quad$ SBR $i$-th species mass fraction [-]

$\rho$ Density in the PFR $\left[\mathrm{kg} \mathrm{m}^{-3}\right]$

$\rho^{*} \quad$ Density in th SBR $\left[\mathrm{kg} \mathrm{m}^{-3}\right]$

$\tau$ Residence time [s] 


\section{$\theta \quad$ Elapsed time $[\mathrm{s}]$}

\section{Other symbols}

$0 \quad$ Initial value (subscript)

(k) Diol synthesis reactor section (superscript)

$\hat{\imath}$ Dimensionless quantity .

\section{References}

[1] F. Maestri, R. Rota, Kinetic-free safe optimization of a semibatch runaway reaction: the nitration of 4-chloro benzotrifluoride, Ind. Eng. Chem. Res. 55 (2016) 12786-12794.

[2] S. Copelli, M. Derudi, J. Sempere, E. Serra, A. Lunghi, C. Pasturenzi, R. Rota, Emulsion polymerization of vinyl acetate: Safe optimization of a hazardous complex process, J. Hazard. Mater. 192 (2011) 8-17.

[3] Z. Anxionnaz, M. Cabassud, C. Gourdon, P. Tochon, Transposition of an exothermic reaction from a batch reactor to an intensified continuous one, Heat Transfer Eng. 31 (9) (2010) 788-797.

[4] C. H. Phillips, G. Lauschke, H. Peerhossaini, Intensification of batch chemical processes by using integrated chemical reactor-heat exchangers, Appl. Therm. Eng. 17 (8-10) (1997) 809-824.

[5] R. J. Minari, L. M. Gugliotta, J. R. Vega, G. R. Meira, Continuous emulsion copolymerization of acrylonitrile and butadiene. computer simulation study for improving the rubber quality and increasing production, Comput. Chem. Eng. 31 (2007) 1073-1080.

[6] S. Ferrouillat, P. Tochon, D. Della Valle, H. Peerhossaini, Open loop thermal control of exothermal chemical reactions in multifunctional heat exchangers, Int. J. Heat Mass Transfer 49 (2006) 2479-2490.

[7] K. Rossow, P. Bröge, F. G. Lüth, P. Joy, A. Mhamdi, A. Mitsos, H.-U. Moritz, W. Pauer, Transfer of emulsion polymerization of styrene and n-butyl acrylate from semi-batch to continuous tubular reactor, Macromol. React. Eng. 10 (2016) $324-338$.

[8] D. Kohlmann, M.-C. Chevrel, S. Hoppe, D. Meimaroglou, D. Chapron, P. Bourson, C. Schwede, W. Loth, A. Stammer, J. Wilson, P. Ferlin, L. Falk, S. Engell, A. Durand, Modular, flexible, and continuous plant for radical polymerization in aqueous solution, Macromol. React. Eng. 10 (2016) 339-353.

[9] T. Goerke, D. Kohlmann, S. Engell, Transfer of semibatch processes to continuous processes with side injections - opportunities and limitations, Macromol. React. Eng. 10 (2016) 364-388.

[10] D. Meimaroglou, M.-C. Chevrel, S. Hoppe, A. Durand, L. Falk, J. Wilson, P. Ferlin, On the modeling of acrylic acid copolymerization in an aqueous solution: A modular, integrated approach, Macromol. React. Eng. 10 (2016) 389-405.

[11] R. Hashemi, D. Kohlmann, S. Engell, Optimizing control and state estimation of a continuous polymerization process in a tubular reactor with multiple side-streams, Macromol. React. Eng. 10 (2016) 415-434.

[12] J. Haber, B. Jiang, T. Maeder, A. Renken, L. Kiwi-Minsker, Multi-injection microstructured reactor for intensification of fast exothermic reactions: proof of concept, Green Process. Synth. 2 (2013) 435-449.

[13] Z. Anxionnaz, M. Cabassud, C. Gourdon, P. Tochon, Heat exchanger/reactors (hex reactors): Concepts, technologies: State-of-the-art, Chem. Eng. Process. 47 (2008) 2029-2050.

[14] T. N. Zwietering, The degree of mixing in continuous flow systems, Chem. Eng. Sci. 11 (1) (1959) 1-15. 
[15] D. R. Bassett, K. L. Hoy, Nonuniform Emulsion Polymers, ACS Publications, 1981, Ch. 23 , pp. $371-387$.

[16] J. C. De La Cal, A. Echevarria, G. R. Meira, J. M. Asua, Minimum-time strategy to produce nonuniform emulsion copolymers. i. theory, J. Appl. Polym. Sci. 57 (9) (1995) 1063-1074.

[17] G. R. Gavalas, The long chain approximation in free radical reaction systems, Chem. Eng. Sci. 21 (2) (1966) $133-142$.

[18] H. Tobita, A. E. Hamielec, Kinetics of free-radical copolymerization: the pseudo-kinetic rate constant method, Polymer 32 (14) (1991) 2641-2647.

[19] F. R. Mayo, F. M. Lewis, Copolymerization. I. A basis for comparing the behavior of monomers in copolymerization; the copolymerization of styrene and methyl methacrylate, J. Am. Chem. Soc. 66 (9) (1944) $1594-1601$.

[20] G. Bringmann, M. Breuning, P. Henschel, J. Hinrichs, Asymmetric synthesis of (M)-2-hydroxymethyl-1-(2-hydroxy-4,6dimethylphenyl)naphthalene via a configurationally unstable biaryl lactone, Org. Synth. 79 (2002) 72. 


\title{
From semi-batch to continuous tubular reactors: a kinetics-free approach
}

\author{
Federico Florit ${ }^{\mathrm{a}}$, Valentina Busini ${ }^{\mathrm{a}}$, Giuseppe Storti $^{\mathrm{b}}$, Renato Rota ${ }^{\mathrm{a}, *}$ \\ ${ }^{a}$ Politecnico di Milano, Dipartimento di Chimica, Materiali e Ingegneria Chimica G. Natta, via Mancinelli 7, 20131, \\ Milano, Italy \\ ${ }^{b}$ ETH Zürich, Department of Chemistry and Applied Biosciences, Vladimir-Prelog-Weg 1-5/10, HCI F 125, 8093 Zürich, \\ Switzerland
}

\begin{abstract}
A methodology, which does not require any kinetic information, for the rigorous transformation of an isothermal, homogeneous semi-batch process into an equivalent continuous side-fed tubular reactor was developed. Once the semi-batch process parameters are known, the proposed methodology allows for easily defining all the process parameters of a side-fed tubular reactor that guarantees the same performances as the original semi-batch process, in terms of conversion and product characteristics.

Two different case studies were selected to investigate the potential of the proposed approach: a copolymer synthesis and the production of a fine chemical, clearly showing the need of a rigorous transformation of the semi-batch process into the continuous one since productivity and product quality are strongly affected by the feeding policy.
\end{abstract}

Keywords: semi-batch to continuous, process intensification, kinetics-free, tubular reactor

\section{Introduction}

Fine chemicals and specialties are generally produced via discontinuous or semi-continuous processes. This is mainly due either to discontinuous market needs (seasonal campaigns), which require a high degree of flexibility in the synthesis, or to complex and dangerous reaction processes, which are carried out in semi-batch reactors, SBRs, for safety reason [1, 2].

An effective way to reduce costs and enhance reproducibility of such processes is to transform discontinuous ones into their continuous counterpart. This way reactor volumes are usually reduced, the intrinsic process safety is increased thanks to the lower hold-up, and investment costs are also decreased [3]. Moreover, overall process times can be reduced and more constant quality is achieved, with a general decrease in

\footnotetext{
*Corresponding author

Email address: renato.rota@polimi.it (Renato Rota)
} 
operative costs [4]. This is the framework of the so called process intensification. Transition from discontinuous to continuous processes is also motivated by the increasing demand of specialty products. Higher production volumes would require more complex and expensive discontinuous equipment, making engineers more prone to consider continuous systems.

Straightforward choices for continuous reactors are mixed vessels (approximating the behaviour of Continuous Stirred Tank Reactors, CSTRs) [5] or tubular reactors (aiming at Plug Flow Reactors, PFRs) [3]. This last configuration also benefits better heat exchange due to higher heat transfer area/volume ratios [6]; as a consequence, it is possible to run reactions in more severe conditions leading to an increase in product quantity/quality, while keeping the process safe. Mixed vessels for continuous synthesis are well investigated and find several industrial applications. On the contrary, only few works analyse the same problem in tubular reactors. Roughly speaking, four main research areas for continuous syntheses can be identified: classical PFR-like reactors [6, 7], modular systems [8-11], microreactors [12] and other reactors [13].

Ferrouillant et al. [6] have studied the improved heat removal in a multifunctional heat exchanger running an exothermic reaction. Rossow et al. [7] have designed a continuous copolymerisation PFR-like reactor from a process carried out in a SBR. Kohlmann et al. [8], Goerke et al. [9] and Hashemi et al. [11] have developed a continuous modular tubular reactor with intermediate injections for a copolymerisation reaction, analysing its feasibility with respect to the original SBR process and proposing also an optimised control system. Meimaroglou et al. [10] used a series of tubular reactors in a modular system to run a solution copolymerisation varying process conditions. Haber et al. [12] have analysed the behaviour of a microstructured tubular reactor running a quasi-instantaneous exothermic reaction. Anxionnaz et al. [13] reviewed different configurations and geometries for process intensification in heat exchanger reactors. All previous works succeeded in finding a specific condition for carrying out the desired process in a given reactor, but seem to lack a sense of generality, being the reported approach limited to the particular process under examination.

This work focuses on continuous side-fed tubular reactors. In particular a PFR with continuous side injections, similar to the one proposed by Zwietering [14], was considered. Given a reaction process effectively carried out in a semi-batch reactor with an optimised recipe, the primary aim was to define the feeding policy for the side injections into the Zwietering-like tubular reactor which allows to reproduce exactly the SBR behaviour without knowing the reaction kinetics. This way, the operating conditions of a tubular reactor having the same efficiency as the original SBR in terms of conversion and selectivity are identified. On the 
other hand, the process productivity remains as degree of freedom and, therefore, can be increased.

\section{Model formulation}

\subsection{Dimensional equations}

Let us consider a generic industrial reaction process through which a chemical species is produced using an optimised recipe in SBR. Figure 1 shows the scheme of such SBR, which is initially charged with a known quantity of each species, $m_{i, 0}$, and fed with a mass flowrate of each component, $F_{i}(t),(i=1, \ldots, N C)$. The reactor is assumed to be well mixed and to operate at isothermal conditions.

Our aim is to transform this discontinuous process into a continuous, steady-state one, using a tubular reactor with side feed injections (Figure 2) and constant temperature (equal to that of the SBR). The key question to be answered is how to distribute the side feed injections to obtain the same performances (in terms of conversion and selectivity) as the original industrial process carried out in SBR.

\subsubsection{SBR governing equations}

The original SBR can be modelled using the conventional conservation equations, considering a process duration equal to $t_{\text {proc }}$. The mass balance equations in terms of mass fractions are:

$$
\frac{d \omega_{i}^{*}}{d t}=\frac{F_{i}(t)}{m}-\omega_{i}^{*} \frac{F(t)}{m}+\frac{\dot{\Omega}_{i}\left(\underline{\omega}^{*}\right)}{\rho^{*}(t)}
$$

where $\omega_{i}^{*}$ is the mass fraction of species $i$ in the SBR, $t$ is the time, $m$ is the total mass, $\dot{\Omega}_{i}$ is species $i$ massive reaction rate, and $\rho^{*}$ is the density of the reactive mixture.

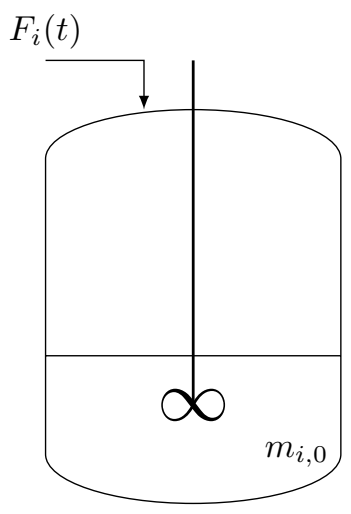

Figure 1: Semi-batch reactor. $m_{i, 0}[\mathrm{~kg}]$ is the initially charged amount of species $i, F_{i}(t)\left[\mathrm{kg} \mathrm{s}^{-1}\right]$ is the feed rate of species $i$. 


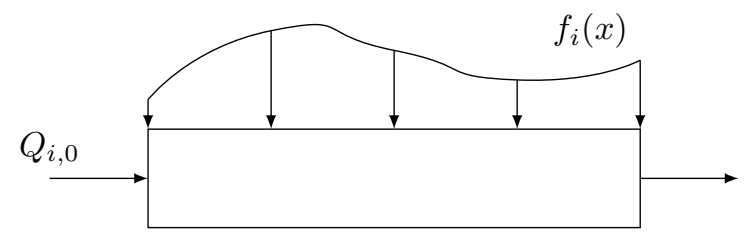

Figure 2: Tubular reactor with distributed side feed. $Q_{i, 0}\left[\mathrm{~kg} \mathrm{~s}^{-1}\right]$ is the inlet flow rate of species $i, f_{i}(x)\left[\mathrm{kg} \mathrm{m}^{-1} \mathrm{~s}^{-1}\right]$ is the specific side feed rate of species $i$.

An additional equation (the overall mass balance) is required to track the total mass increase in time:

$$
\frac{d m}{d t}=F(t)
$$

where

$$
F(t) \stackrel{\text { def }}{=} \sum_{i=1}^{N C} F_{i}(t)
$$

This system of ODEs is completed by the corresponding initial conditions, ICs:

$$
\begin{gathered}
\omega_{i}^{*}(0)=\frac{m_{i, 0}}{m_{0}} \\
m(0)=m_{0}
\end{gathered}
$$

where $m_{0}=\sum_{i=1}^{N C} m_{i, 0}$ is the total initial mass.

Note that the system density, $\rho^{*}$, can be computed through a proper equation of state as a function of composition (and therefore time):

$$
\rho^{*}(t)=\rho\left(\underline{\omega}^{*}(t)\right)
$$

The simplest equation of state that can be used is obviously $\rho^{*}(t)=$ const.

The equations above are used to compute the time evolution of mass fractions, $\omega_{i}^{*}$, total mass, $m$, and overall density, $\rho^{*}$, given the reaction kinetics, $\dot{\Omega}_{i}\left(\underline{\omega}^{*}\right)$, and the feed rates, $F_{i}(t)$. However, it is worth noting that also measurements of the time evolutions of the same quantities (composition, overall mass, and density) in the existing industrial SBR are enough to use the proposed approach.

\subsubsection{Tubular reactor governing equations}

Similar mass balance equations can be written for the tubular reactor with side injections at steady state:

$$
\frac{d \omega_{i}}{d x}=\frac{1}{Q}\left(f_{i}(x)-\omega_{i} f(x)+\dot{\Omega}_{i}(\underline{\omega}) \mathscr{A}(x)\right)
$$


where $\omega_{i}$ is species $i$ mass fraction in the tubular reactor, $x$ is the axial coordinate, $Q$ is the total mass flowrate, $\mathscr{A}$ is the cross sectional area and

$$
f(x) \stackrel{\text { def }}{=} \sum_{i=1}^{N C} f_{i}(x)
$$

The total flowrate changes along the axial coordinate according to the overall mass balance:

$$
\frac{d Q}{d x}=f(x)
$$

Again, the previous equations ask for boundary conditions, BCs:

$$
\begin{gathered}
\omega_{i}(0)=\omega_{i, 0} \\
Q(0)=Q_{0}
\end{gathered}
$$

where $Q_{0}$ and $\omega_{i, 0}$ are the inlet mass flowrate and inlet species $i$ mass fraction, respectively.

Again, an equation of state (the same used to simulate the SBR) is required to evaluate the density profile, $\rho(x)$, as a function of composition:

$$
\rho(x)=\rho(\underline{\omega}(x))
$$

Finally, it is convenient to introduce the elapsed time, $\theta(x)$, defined as the time spent by a generic fluid element to reach the position $x$ after entering the reactor:

$$
\frac{d \theta}{d x}=\frac{\rho(x) \mathscr{A}(x)}{Q(x)}
$$

This equation follows from the definition of the fluid parcel instantaneous velocity from an inertial observer point of view. It also provides the connection between time and space inside the tubular reactor and it will be helpful to build the relationship between the SBR and the Zwietering-like reactor.

The final system involves $(N C+4)$ equations (namely, Equations (7), (8), (9), (12) and (13)) and $(3 N C+7)$ unknowns: $\underline{\omega}(x), \underline{\omega}_{0}, Q(x), Q_{0}, \underline{f}(x), f(x), \theta(x), \rho(x), \mathscr{A}$, and the reactor length $L$. Therefore, $(2 N C+3)$ degrees of freedom need to be specified to make the problem defined. 


\subsubsection{Proposed semi-batch to continuous transformation procedure}

A connection between the SBR and a Zwietering-like reactor can be established forcing the same species mass fraction to be present at a given SBR time $(t)$ and at the corresponding tubular reactor position $(x)$, that is, at the coordinate reached by a fluid element after time $t=\theta(x)$ :

$$
\omega_{i}(x)=\omega_{i}^{*}(\theta(x)) \quad \forall x \in[0, L]
$$

Note that, as a consequence, the same relation holds true for the density, i.e., $\rho(x)=\rho^{*}(\theta(x))$.

Moreover, to ensure the same overall process time in both reactors, the following equation applies:

$$
\theta(L)=t_{\text {proc }}
$$

Equations (14) and (15) enforce $2 N C+1$ additional constraints, being the former valid not only along the reactor, but also at the inlet where the BCs (10) apply (i.e., $\left.\omega_{i}(0)=\omega_{i}^{*}(0)=\frac{m_{i, 0}}{m_{0}}\right)$. Therefore, 2 degrees of freedom remain, which can be saturated by setting arbitrary values of $Q_{0}$ and $\mathscr{A}(x)$. Note that different values of these quantities lead to different values of productivity and length of the tubular reactor.

Equations (7) and (14) together with (13) lead to (note that in the following the dependencies on $x$ are dropped for brevity):

$$
\omega_{i}^{*}(\theta) f+\left.Q \frac{d \omega_{i}^{*}}{d \theta}\right|_{\theta=t} \frac{d \theta}{d x}=\omega_{i}^{*}(\theta) f+\left.\frac{d \omega_{i}^{*}}{d t}\right|_{t=\theta} \rho \mathscr{A}=f_{i}+\dot{\Omega}_{i}\left(\underline{\omega}^{*}(\theta)\right) \mathscr{A}
$$

which, combined with Equation (1), becomes:

$$
\omega_{i}^{*}(\theta)\left(f-\rho \mathscr{A} \frac{F(\theta)}{m(\theta)}\right)=\omega_{i}^{*}(\theta) \sum_{j=1}^{N C}\left(f_{j}-\rho \mathscr{A} \frac{F_{j}(\theta)}{m(\theta)}\right)=f_{i}-\rho \mathscr{A} \frac{F_{i}(\theta)}{m(\theta)}
$$

where, $m(\theta)$ can be obtained from Equation (2) as:

$$
m(\theta)=m_{0}+\int_{0}^{\theta} F(t) d t
$$


Equation (17) leads to a linear system $\underline{\underline{A} y}=\underline{0}$, where

$$
\begin{aligned}
\underline{\underline{A}}= & {\left.\left[\begin{array}{cccc}
\omega_{1}^{*} & \omega_{1}^{*} & \ldots & \omega_{1}^{*} \\
\omega_{2}^{*} & \omega_{2}^{*} & \ldots & \omega_{2}^{*} \\
\vdots & \vdots & \ddots & \vdots \\
\omega_{N C}^{*} & \omega_{N C}^{*} & \ldots & \omega_{N C}^{*}
\end{array}\right]\right|_{t=\theta}-\underline{\underline{I}} } \\
\underline{y} & =\left[\begin{array}{c}
f_{1}-\rho \mathscr{A} \frac{F_{1}(\theta)}{m(\theta)} \\
f_{2}-\rho \mathscr{A} \frac{F_{2}(\theta)}{m(\theta)} \\
\vdots \\
f_{N C}-\rho \mathscr{A} \frac{F_{N C}(\theta)}{m(\theta)}
\end{array}\right]
\end{aligned}
$$

and $\underline{\underline{I}}$ is the identity matrix. Nevertheless, these equations are not linearly independent. In fact, an identity is obtained if all equations are summed up since the stoichiometric condition $\sum_{i=1}^{N C} \omega_{i}^{*}=1$ holds true. It should be noted that at least one unknown is zero: this is the case when a species is neither fed to the SBR nor to the tubular reactor side (for instance, this is true when the species is a product). In this case the solution of the system is the trivial one, $\underline{y}=\underline{0}$, that is:

$$
f_{i}-\rho \mathscr{A} \frac{F_{i}(\theta)}{m(\theta)}=0
$$

The same solution could be obtained even if all species were fed to the SBR since one function $f_{k}(x)$ can be arbitrarily chosen to fulfil the constraint $y_{k}=0$. Therefore, in any case it follows that the solution of the previous linear system of equations reduces to:

$$
f_{i}(x)=\rho \mathscr{A} \frac{F_{i}(\theta(x))}{m(\theta(x))}
$$

This last equation can be recast as:

$$
\frac{f_{i}(x)}{\mathscr{A}(x)}=\frac{F_{i}(\theta(x))}{V(\theta(x))}
$$

where $V(\theta(x))=\frac{m(\theta(x))}{\rho^{*}(\theta(x))}$ is the reaction volume in the SBR at time $t=\theta(x)$. This means that the volume specific mass flowrate must be the same in both the SBR and the Zwietering-like reactor at the same time. Note that the ratio $f_{i}(x)$ to $\mathscr{A}(x)$ has units of flowrate per volume and it represents the feed profile which allows reproducing a process carried out in a SBR by a continuous tubular reactor while keeping the same performances in terms of both conversion and selectivity. 


\subsubsection{Model constitutive equations}

The proposed model for the rigorous transformation of a SBR process into a Zwietering-like tubular reactor process is based on Equations (9), (13), (15) and (21) together with the required boundary conditions, that is:

$$
\left\{\begin{array} { l } 
{ \frac { d Q } { d x } = \sum _ { i = 1 } ^ { N C } f _ { i } ( x ) } \\
{ \frac { d \theta } { d x } = \frac { \rho \mathscr { A } } { Q ( x ) } } \\
{ \theta ( L ) = t _ { \text { proc } } } \\
{ f _ { i } ( x ) = \rho \mathscr { A } \frac { F _ { i } ( \theta ) } { m ( \theta ) } }
\end{array} \quad \left\{\begin{array}{l}
\theta(0)=0 \\
Q(0)=Q_{0}
\end{array}\right.\right.
$$

This is a set of 2 ODEs with Dirichlet BCs and $N C+1$ algebraic relationships. Note that the algebraic equations are uncoupled from the ODEs solution, therefore this system is not DAE. From a numerical point of view, the ODE system must be integrated until the exit condition, $\theta(L)=t_{\text {proc }}$, is met, thus providing the tubular reactor length. Moreover, since the ODEs solution provides the $\theta(x)$ relation, the required side feed rates $f_{i}(x)$ can be easily computed.

\subsection{Dimensionless equations}

Dimensionless equations can be obtained from the aforementioned ones by defining the following dimensionless variables:

$$
\begin{aligned}
& \hat{x} \stackrel{\text { def }}{=} \frac{x}{L} \rightarrow \quad d x=L d \hat{x} \\
& \hat{t} \stackrel{\text { def }}{=} \frac{t}{t_{\text {proc }}} \rightarrow d t=t_{\text {proc }} d \hat{t} \\
& \hat{Q}(\hat{x}) \stackrel{\text { deff }}{=} \frac{Q(L \hat{x})}{Q_{0}} \rightarrow d Q=Q_{0} d \hat{Q} \\
& \hat{f}_{i}(\hat{x}) \stackrel{\text { def }}{=} \frac{L}{Q_{0}} f_{i}(L \hat{x}) \\
& \hat{\rho}(\hat{x}) \stackrel{\text { def }}{=} \frac{\rho(L \hat{x})}{\rho_{0}} \\
& \hat{\rho}^{*}(\hat{t}) \stackrel{\text { def }}{=} \frac{\rho^{*}\left(t_{\text {proc }} \hat{t}\right)}{\rho_{0}} \\
& \hat{\mathscr{A}}(\hat{x}) \stackrel{\text { def }}{=} \frac{\mathscr{A}(L \hat{x})}{\mathscr{A}_{0}}
\end{aligned}
$$


It should be noted that $\hat{\rho}^{*}(\hat{\theta})=\hat{\rho}(\hat{x})$ since density depends on the composition only, as a consequence of Equation (14). Using these definitions, Equations (9), (13) and (21) become:

$$
\begin{gathered}
\frac{d \hat{Q}}{d \hat{x}}=\hat{f} \\
\frac{d \hat{\theta}}{d \hat{x}}=\frac{\frac{L \rho_{0} \mathscr{A}_{0}}{t_{\text {proc }} Q_{0}} \hat{\rho}^{*}(\hat{\theta}) \hat{\mathscr{A}}(\hat{x})}{\hat{Q}}=\frac{K \hat{\rho}^{*}(\hat{\theta}) \hat{\mathscr{A}}(\hat{x})}{\hat{Q}} \\
\hat{f}_{i}=\frac{L \rho_{0} \mathscr{A}_{0}}{t_{\text {proc }} Q_{0}} \hat{\rho}^{*}(\hat{\theta}) \hat{\mathscr{A}}(\hat{x}) \frac{F_{i}(\theta) t_{\text {proc }}}{m(\theta)}=K \hat{\rho}^{*}(\hat{\theta}) \hat{\mathscr{A}}(\hat{x}) \frac{F_{i}(\theta) t_{\text {proc }}}{m(\theta)}
\end{gathered}
$$

where $\hat{Q}(0)=1, \hat{\theta}(0)=0$ and

$$
K \stackrel{\text { def }}{=} \frac{L \rho_{0} \mathscr{A}_{0}}{t_{\text {proc }} Q_{0}}=\frac{\frac{L}{\mathrm{v}_{0}}}{t_{\text {proc }}}=\frac{\tau_{0}}{t_{\text {proc }}}=\frac{L}{L_{0}}
$$

is a dimensionless number expressing the ratio of the initial residence time with respect to the overall process time, which can also be considered as a dimensionless tubular reactor length. Here $\mathrm{v}_{0}=\frac{Q_{0}}{\rho_{0} \mathscr{A}_{0}}$ is the initial velocity, $\tau_{0}=\frac{L}{\mathrm{v}_{0}}$ is a formal initial residence time (that is, the one computed without accounting for the side feed) and $L_{0}=\frac{\mathrm{v}_{0}}{t_{\text {proc }}}$ is the reactor length computed without considering the side injections.

By further defining

$$
\hat{F}_{i}(\hat{\theta}) \stackrel{\text { def }}{=} F_{i}\left(t_{\text {proc }} \hat{\theta}\right) \frac{t_{\text {proc }}}{m_{0}}
$$

one gets

$$
\begin{aligned}
\frac{F_{i}(\theta)}{m(\theta)} & =\frac{F_{i}(\theta)}{m_{0}+\int_{0}^{\theta} F(t) d t}= \\
& =\frac{1}{t_{\text {proc }}} \frac{\hat{F}_{i}(\hat{\theta})}{1+\int_{0}^{\hat{\theta}} \hat{F}(\hat{t}) d \hat{t}} \stackrel{\text { def }}{=} \frac{\hat{\gamma}_{i}(\hat{\theta})}{t_{\text {proc }}}
\end{aligned}
$$

and Equation (25) becomes:

$$
\frac{d \hat{Q}}{d \hat{x}}=\sum_{i=1}^{N C} \hat{\gamma}_{i}(\hat{\theta})
$$

By applying the chain rule and using Equation (26) it is possible to obtain:

$$
\frac{d \hat{Q}}{d \hat{\theta}}=K \hat{\rho}^{*}(\hat{\theta}) \hat{\mathscr{A}}(\hat{x}) \sum_{i=1}^{N C} \hat{\gamma}_{i}(\hat{\theta})\left(\frac{d \hat{\theta}}{d \hat{x}}\right)^{-1}=\hat{Q} \sum_{i=1}^{N C} \hat{\gamma}_{i}(\hat{\theta})
$$


This equation can be integrated, leading to

$$
\begin{aligned}
\hat{Q}(\hat{\theta}) & =\exp \left(\int_{0}^{\hat{\theta}} \sum_{i=1}^{N C} \hat{\gamma}_{i}(\hat{t}) d \hat{t}\right)= \\
& =1+\int_{0}^{\hat{\theta}} \hat{F}(\hat{t}) d \hat{t}
\end{aligned}
$$

where

$$
\hat{F}(\hat{t})=\sum_{i=1}^{N C} \hat{F}_{i}(\hat{t})
$$

which allows solving Equation (26) as:

$$
\int_{0}^{\hat{\theta}} \frac{\hat{Q}(\hat{t})}{\hat{\rho}^{*}(\hat{t})} d \hat{t}=K \int_{0}^{\hat{x}} \hat{\mathscr{A}}(\hat{\xi}) d \hat{\xi}
$$

to obtain the function $\hat{\theta}(\hat{x})$.

$K$ value can be found by using the dimensionless Equation (15)

$$
\hat{\theta}(1)=1
$$

together with Equation (35)

$$
K=\frac{\int_{0}^{1} \frac{\hat{Q}(\hat{\theta})}{\hat{\rho}^{*}(\hat{\theta})} d \hat{\theta}}{\int_{0}^{1} \hat{\mathscr{A}}(\hat{x}) d \hat{x}}
$$

Consequently, Equation (27) becomes:

$$
\hat{f}_{i}(\hat{x})=K \hat{\rho}^{*}(\hat{\theta}) \hat{\mathscr{A}}(\hat{x}) \frac{\hat{F}_{i}(\hat{\theta})}{\hat{Q}(\hat{\theta})}
$$

The solution of this problem is guaranteed to exist and to be unique by the Cauchy-Lipschitz theorem. In fact the problem consists in the solution of a Cauchy problem: the integrand function $\hat{\theta}(\hat{x})$ is Lipschitz continuous on $[0,1]$ as it has bounded first derivative, therefore satisfying the theorem conditions.

Note that the trivial case with constant density and cross sectional area leads to the simpler equation:

$$
K=\int_{0}^{1} \hat{Q}(\hat{\theta}) d \hat{\theta}
$$

The general algorithm used to solve this problem is the following: 
1. assign a value to $\mathscr{A}(\hat{x})$ and $Q_{0}$;

2. from the SBR recipe, compute $\hat{\rho}^{*}(\hat{t}), \hat{F}_{i}(\hat{t})$, and $\hat{F}(\hat{t})$ through Equations (24), (29) and (34);

3. compute $\hat{Q}(\hat{\theta})$ through Equation (33);

4. compute $K$ through Equation (37);

5. solve ODE (26) to compute $\hat{\theta}(\hat{x})$;

6. compute $\hat{f}_{i}(\hat{x})$ using Equation (38);

7. find the tubular reactor length using Equation (28).

From these dimensionless values, dimensional values can be easily computed through their definitions (24).

An important feature of the proposed procedure is now evident: no kinetic information is required once an effective recipe is available. In other words, some measurements on the industrial SBR (namely: $\rho^{*}(t), \underline{F}(t)$, and $m_{0}$ ) suffice to identify all the parameters of a side fed tubular reactor which is able to achieve the same product quality (at least in terms of conversion and selectivity) of an existing SBR industrial plant.

\section{Parametric analysis}

The proposed procedure guarantees a rigorous transformation of a SBR based process to a continuous one carried out in a Zwietering-like tubular reactor. However, also more trivial procedures, based on rulesof-thumb arising from engineering intuition, could be guessed. To highlight the different results (in terms of conversion and selectivity) obtained using the proposed methodology or a more intuitive side feed injection policy, a simple case study was investigated. Density and cross sectional area are assumed constant for the sake of simplicity and a second-order process reaction is considered:

$$
A+B \rightarrow C \quad r_{1}=k_{1} c_{A} c_{B}
$$

along with an undesired first-order side reaction consuming the desired product, $C$ :

$$
C \rightarrow D \quad r_{2}=k_{2} c_{C}
$$

According to the SBR recipe, a given amount of the main reactant $B, m_{0}$, has to be initially loaded in the reactor, while the coreactant $A$ is fed at constant flowrate, $F_{A 0}$. For this simple case study, an analytical solution can be found by applying the aforementioned procedure: 
1. arbitrary values can be chosen for both $\mathscr{A}_{0}$ and $Q_{0}$; however, being the cross sectional area constant, it follows that:

$$
\hat{\mathscr{A}}=1
$$

2. $\hat{\rho}^{*}(\hat{t}), \hat{F}_{i}(\hat{t})$ and $\hat{F}(\hat{t})$ are computed using Equations (24), (29) and (34), resulting in the following constant values:

$$
\begin{gathered}
\hat{\rho}^{*}=1 \\
\hat{F}=\hat{F}_{A}=\frac{t_{\text {proc }}}{m_{0}} F_{A 0} \quad \hat{F}_{B}=\hat{F}_{C}=\hat{F}_{D}=0
\end{gathered}
$$

3. $\hat{Q}(\hat{\theta})$ is computed through Equation (33), leading to:

$$
\hat{Q}(\hat{\theta})=1+\hat{F} \hat{\theta}
$$

4. $K$ is then computed using Equation (37):

$$
K=1+\frac{\hat{F}}{2}
$$

5. the ODE (26) can be integrated analytically to give the functional dependence $\hat{\theta}(\hat{x})$ :

$$
\hat{\theta}(\hat{x})=\frac{1}{\hat{F}}(\sqrt{1+2 K \hat{F} \hat{x}}-1)
$$

6. $\hat{f}_{A}(\hat{x})$ is computed using Equation (38) (note that only the coreactant $A$ is fed to the SBR and therefore injected laterally in the tubular reactor):

$$
\hat{f}_{A}(\hat{x})=\frac{K \hat{F}}{\sqrt{1+2 K \hat{F} \hat{x}}}
$$

7. finally, $\hat{f}_{A}(\hat{x})$ is made dimensional using Equation (38) and the tubular reactor length is found using Equation (28)

$$
\begin{aligned}
& f_{A}(x)=\frac{\rho_{0} \mathscr{A}_{0} F_{A 0}}{m_{0} \sqrt{1+2 \frac{\rho_{0} \mathscr{A}_{0} F_{A 0}}{m_{0} Q_{0}} x}} \\
& L=\left(1+\frac{F_{A 0} t_{\text {proc }}}{2 m_{0}}\right) \frac{Q_{0} t_{p r o c}}{\rho_{0} \mathscr{A}_{0}}
\end{aligned}
$$

As previously stated, it is important noting that no chemical kinetics information was used to evaluate $f_{A}(x)$ 
and $L$.

This solution can be compared with an approximate solution derived from a more intuitive transformation involving constant side feedrate, $f_{A 0}$, all along the entire tubular reactor. For instance, this constant side feedrate can be computed using the following similarity relationship between tubular reactor flowrates and SBR masses:

$$
\frac{f_{A 0} L}{Q_{0}}=\frac{F_{A 0} t_{\text {proc }}}{m_{0}}
$$

Moreover, the mean residence time in the tubular reactor can be set equal to the SBR process time as follows:

$$
\frac{\rho \mathscr{A} L}{Q_{0}+\frac{f_{A 0} L}{2}}=t_{\text {proc }}
$$

Also the use of these rules-of-thumb leads to analytical relations for both $f_{A 0}$ and $L$ :

$$
\begin{gathered}
f_{A 0}=\frac{\rho_{0} \mathscr{A}_{0} F_{A 0}}{m_{0}\left(1+\frac{F_{A 0} t_{\text {proc }}}{2 m_{0}}\right)} \\
L=\left(1+\frac{F_{A 0} t_{\text {proc }}}{2 m_{0}}\right) \frac{Q_{0} t_{\text {proc }}}{\rho_{0} \mathscr{A}_{0}}
\end{gathered}
$$

The two different lateral feed policies are compared in Figure 3 for different values of the parameter $\hat{F}$, which represents the amount of coreactant fed to the SBR with respect to the initially loaded amount of reactants. At increasing values of $\hat{F}$, the variation of the required lateral feed with the axial coordinate increases and, therefore, the differences between the two feed laws increase as well.

In particular, it is worthwhile noting that the constant feed policy of the SBR results in a non-constant lateral feed flowrate along the axial coordinate of the tubular reactor. This behaviour arises from the constraint (22): being $\mathscr{A}$ and $F_{A}$ constant in this case study, the time variation of the SBR reaction volume results in a $f_{A}$ change along the axial coordinate of the tubular reactor. Note that, in spite of the linear variation of the volume in time, the axial variation of $f_{A}$ is not linear due to the non-linear dependence of $\theta$ on $x$.

The practical impact of the different feed policies can be estimated by integrating the mass balances (Equations (7) and (9)) for both constant and variable lateral feed policies. Then, the outlet flowrates of the desired product $C$ computed using the process parameters summarised in Tables 1 and 2 are compared through their percentage relative difference, defined as:

$$
\Delta Q_{C}=\left|\frac{Q_{C}-Q_{C}^{c o n s t}}{Q_{C}}\right|_{x=L} \cdot 100
$$




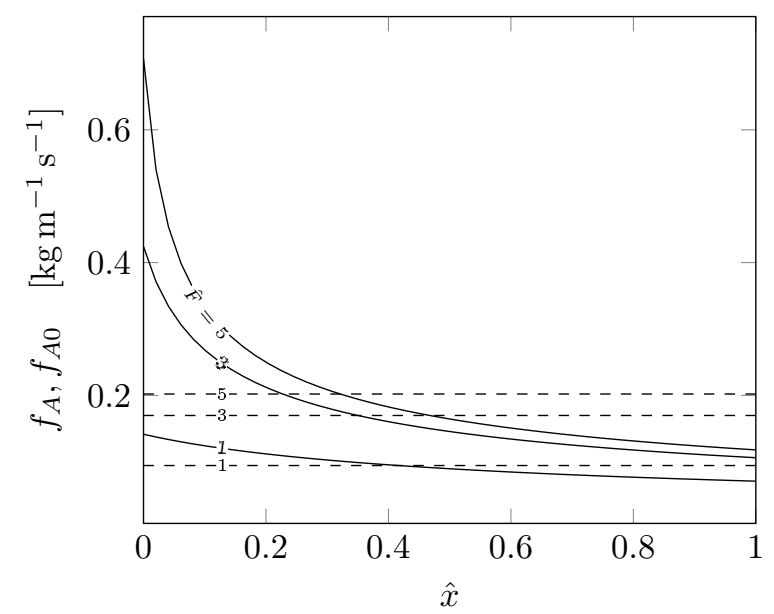

Figure 3: Side feed flowrate along the dimensionless axial coordinate for the rigorous (solid line) and for the constant lateral feed (dashed line) transformations. Curves are parametrised according to the dimensionless SBR feedrate, $\hat{F}$.

Table 1: Process data for parametric analysis simulations.

\begin{tabular}{ll}
\hline$M W_{A}=M W_{B}$ & $20 \mathrm{~kg} \mathrm{kmol}^{-1}$ \\
$M W_{C}=M W_{D}$ & $40 \mathrm{~kg} \mathrm{kmol}^{-1}$ \\
$m_{0}$ & $1000 \mathrm{~kg}$ \\
$t_{\text {proc }}$ & $500 \mathrm{~s}$ \\
$\rho$ & $1000 \mathrm{~kg} \mathrm{~m}^{-3}$ \\
$Q_{0}$ & $1 \mathrm{~kg} \mathrm{~s}^{-1}$ \\
$\mathscr{A}$ & $0.07 \mathrm{~m}^{2}$ \\
\hline
\end{tabular}

in Figure 4 as a function of the dimensionless SBR feed rate, $\hat{F}$, parametrised according to the kinetic constant values of the two reactions, $k_{1}$ and $k_{2} . Q_{C}$ is the flowrate computed using the rigorous transformation policy, while $Q_{C}^{\text {const }}$ is that computed using constant lateral feed flowrate. We can see that large differences arise, clearly indicating that the proposed approach is more effective than simpler rules-of-thumb in the general case.

Another comparison was performed between the continuous lateral feed injection and a discrete one, to investigate the possibility of implementing the proposed procedure with a series of finite additions. This was done by dividing the computed reactor length in $N_{d}$ equal parts, each having a lateral feed injection at

Table 2: Reaction rate constants for the selected cases.

\begin{tabular}{ccc}
\hline & $k_{1} \cdot 10^{6}$ & $k_{2} \cdot 10^{4}$ \\
& {$\left[\frac{\mathrm{m}^{3}}{\mathrm{kmols}}\right]$} & {$\left[\mathrm{s}^{-1}\right]$} \\
$\mathrm{a}$ & 1 & 1 \\
$\mathrm{~b}$ & 1 & 100 \\
$\mathrm{c}$ & 100 & 1 \\
$\mathrm{~d}$ & 100 & 100 \\
\hline
\end{tabular}




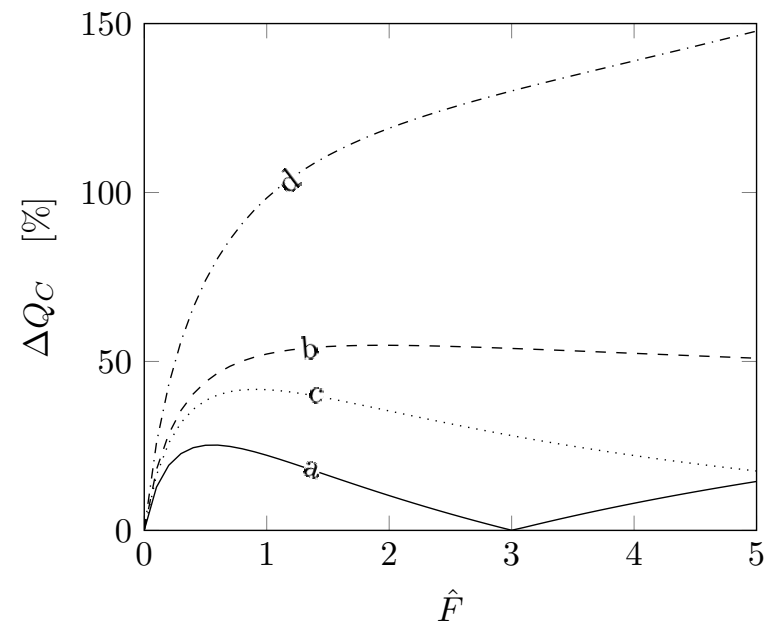

Figure 4: Percentage relative difference between outlet flowrates of species $C$ (Equation (55)) computed using the constant side feed policy and the rigorous one. Curves are parametrised according to the kinetic constants, as from Table 2.

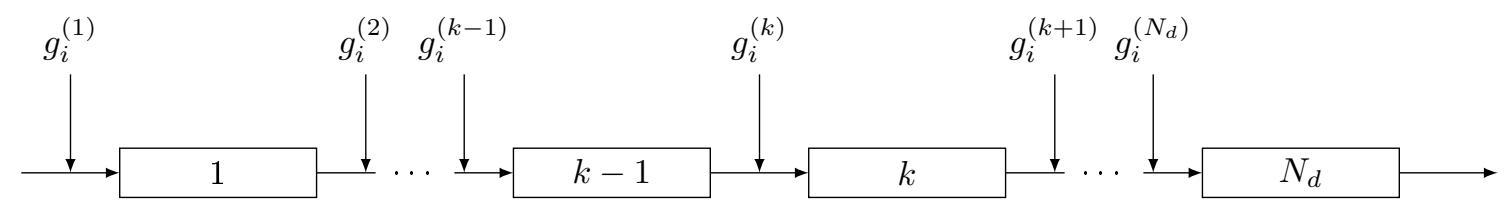

Figure 5: Approximation of the continuous lateral feed injection, $f_{i}(x)$, with $N_{d}$ discrete side injections, $g_{i}^{(k)}$.

its beginning, with a mass flowrate equal to

$$
g_{i}^{(k)}=\int_{\frac{L}{N_{d}}(k-1)}^{\frac{L}{N_{d}} k} f_{i}(x) d x \quad k=1, \ldots, N_{d}
$$

as shown in Figure 5; obviously, $L$ and $f_{i}(x)$ values are computed using the rigorous approach.

Modelling such reactor configuration requires the solution of $N_{d} \cdot N C$ PFR mass balance equations:

$$
\frac{d Q_{i}^{(k)}}{d x}=\dot{\Omega}_{i}\left(\underline{\omega}^{(k)}\right)
$$

where $Q_{i}^{k}$ is the mass flowrate of species $i$ along the axial coordinate of the $k$-th section, and

$$
\omega_{i}^{(k)}=\frac{Q_{i}^{(k)}}{\sum_{j=1}^{N C} Q_{j}^{(k)}}
$$

These equations are solved with BCs:

$$
Q_{i}^{(k)}(0)=Q_{i, \text { out }}^{(k-1)}+g_{i}^{(k)}
$$




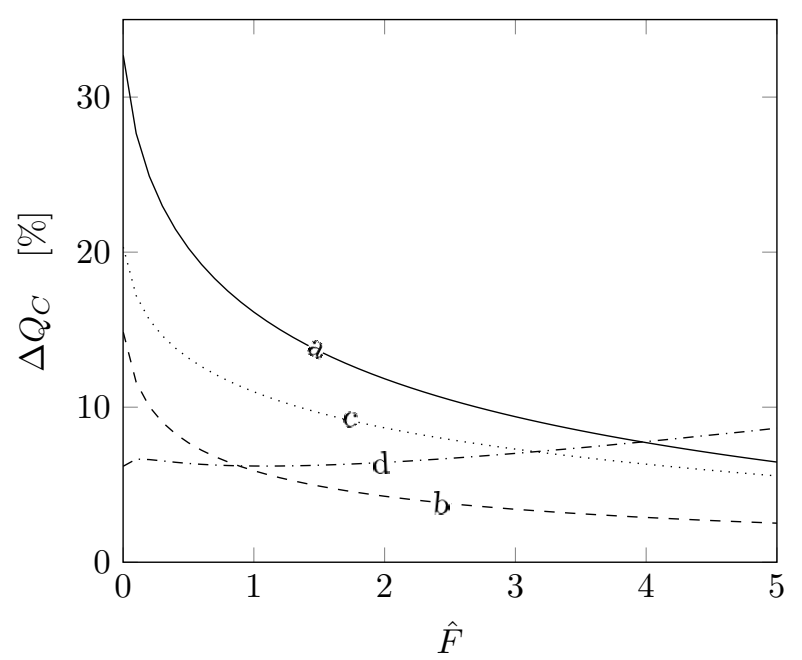

(a) $N_{d}=3$

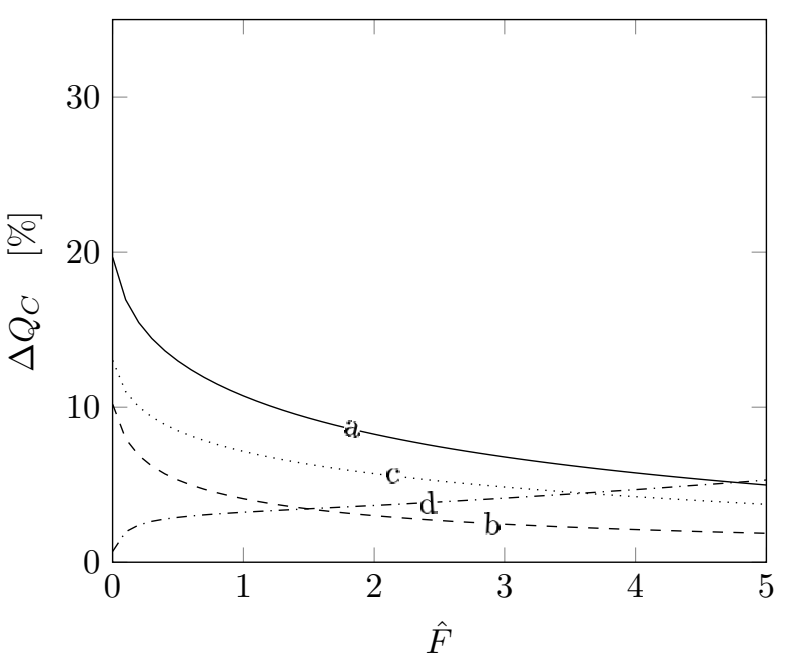

(b) $N_{d}=5$

Figure 6: Percentage relative difference between outlet flowrates for species $C$ (Equation (55)) using the discrete side feed policy with respect to the rigorous one. Curves are parametrised according to the kinetic constants, as from Table 2.

where $Q_{i, \text { out }}^{(k-1)}$ is the flowrate of species $i$ at the outlet of the previous $(k-1)$ section, defined as:

$$
Q_{i, \text { out }}^{(k-1)}= \begin{cases}Q_{0} \omega_{i, 0} & k=1 \\ \left.Q_{i}^{(k-1)}\right|_{x=\frac{L}{N_{d}}} & k>1\end{cases}
$$

Figure 6 compares the continuous and discrete feed policies for different values of the kinetic constants in terms of $\Delta Q_{C}$ as defined by Equation (55). Even though the differences are not negligible, they are definitely lower than those found using a constant side feed rate policy and obviously decrease by increasing the number of subdivisions, as can be seen from Figures 7 and 8 . The former shows how the maximum difference in productivity changes according to the number of discrete feeds, for any analysed dimensionless feedrate and couple of kinetic constants. The latter depicts $C$ mass fractions according to the number of feed locations for an example case. Differences decrease super-linearly between the ideal continuous feed policy and the discrete one at increasing number of feed points. If a maximum deviation of $5 \%$ can be accepted, a critical value (above which deviations are smaller) of $N_{d}=20$ is obtained. 


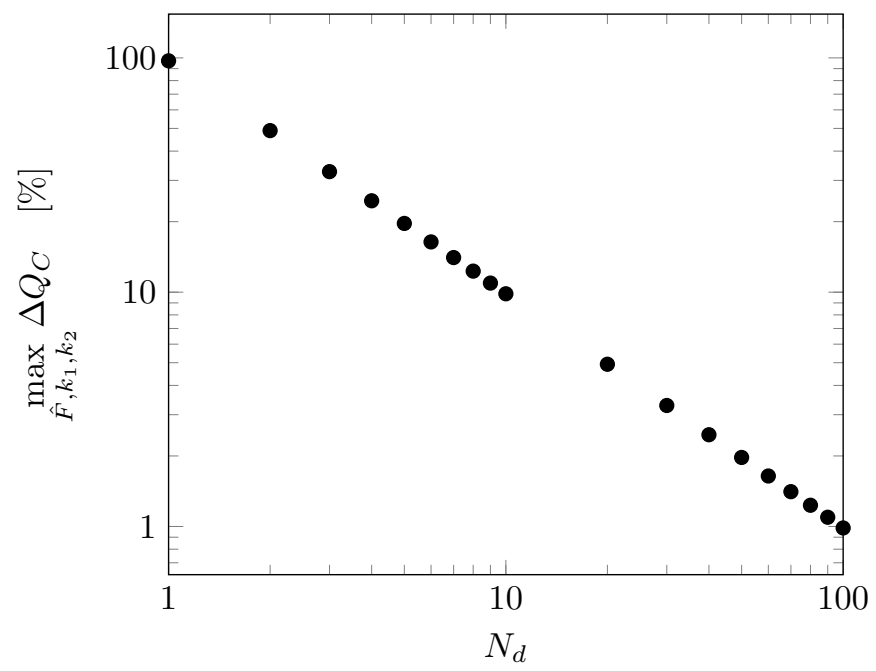

Figure 7: Maximum relative difference between outlet flowrates for species $C$ using the discrete side feed policy with respect to the rigorous one as function of the number of discrete feeds.

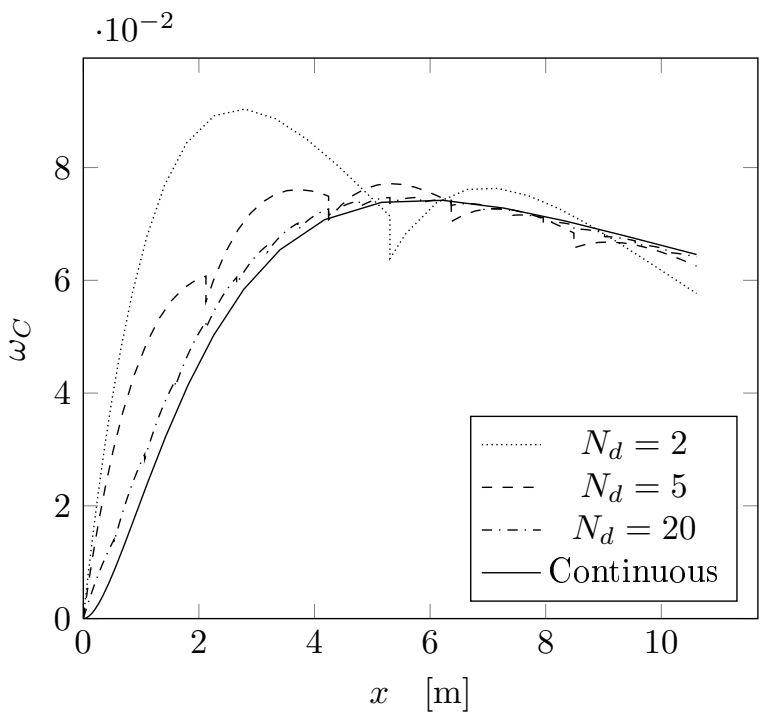

Figure 8: $C$ mass fraction along the axial coordinate where $\hat{F}=1, \quad k_{1}=1 \times 10^{-4} \mathrm{~m}^{3} \mathrm{kmol}^{-1} \mathrm{~s}^{-1}$ and $k_{2}=$ $1 \times 10^{-2} \mathrm{~m}^{3} \mathrm{kmol}^{-1} \mathrm{~s}^{-1}$. Curves are parametrised according to the number of discrete feeds. 


\section{Case studies}

The potentials of the proposed approach were investigated through two case studies, representative of two relevant classes of industrial processes: production of copolymers and production of fine chemicals.

\subsection{Copolymerisation}

\subsubsection{SBR feed policy design}

The feedrate of a copolymerisation SBR can be designed to obtain a given product composition by means of the well known power feed policy $[15,16]$. According to this feed policy, the rate between the residual amounts of the two monomers ( $A$, the most reactive, and $B$, the least reactive) is kept constant during the entire process, thus enabling the production of a copolymer at constant composition, i.e. with the desired monomer $i$ mass fraction, $\phi_{i}^{w}$. A typical reaction scheme for a radical copolymerisation process involves initiation, propagation and termination reactions, as summarised in Table 3. According to the power feed policy, $A$ is the coreactant to be fed to the SBR, while the stoichiometric amount of $B$ is charged to the reactor at the beginning of the process. Therefore, molar balances for the two monomers in the SBR are:

$$
\left\{\begin{array}{l}
\frac{d n_{A}}{d t}=\dot{N}_{A}(t)+R_{A} V \\
\frac{d n_{B}}{d t}=R_{B} V
\end{array}\right.
$$

where $n_{i}(t)$ is the molar quantity of species $i, \dot{N}_{A}$ is the feedrate of $A$ to the SBR, $R_{i}=-k_{p i}^{*} c_{R} \bullet c_{i}$ is species $i$ molar production rate, $c_{i}$ is species $i$ molar concentration and $c_{R}$. is the overall concentration of active chains, given by the quasi-steady-state formula $\sqrt{\frac{R_{I}}{k_{t}^{*}}}$. Assuming long chains approximation (LCA) [17] and the pseudo-kinetic approach [18], the expressions of effective rate coefficients (indicated by the superscript $*$ ) can be readily evaluated as a function of the actual monomer mixture composition as summarised in Table 4.

The analytical solution of these equations is:

$$
\begin{gathered}
n_{B}(t)=\frac{n_{B, 0}}{n_{A, 0}} n_{A}(t)=n_{B, 0} e^{-\tilde{k}_{d} t} \\
\dot{N}_{A}(t)=\dot{N}_{A, 0} e^{-\tilde{k}_{d} t}
\end{gathered}
$$

where $n_{A, 0}$ and $n_{B, 0}$ can be obtained from the so called Mayo-Lewis plot [19], which reports monomer $A$ molar fraction of the instantaneously produced copolymer, $\phi_{A}$, as a function of the same monomer molar 
Table 3: Radicalic copolymerisation reaction scheme. All reactions are bimolecular second order, except initiation, which is unimolecular with rate $R_{I}$. $A_{l, m}^{\bullet}$ stands for a chain of $l$ units of $A$ and $m$ units of $B$, where the radical is located on the terminating $A$ unit.

\begin{tabular}{lll}
\hline & Reaction & Rate constant \\
\hline Initiation & $I_{2}+M \rightarrow 2 R^{\bullet}$ & \\
Propagation & $A_{l, m}^{\bullet}+A \rightarrow A_{l+1, m}^{\bullet}$ & $k_{p A A}$ \\
& $A_{l, m}^{\bullet}+B \rightarrow B_{l, m+1}^{\bullet}$ & $k_{p A B}$ \\
& $B_{l, m}^{\bullet}+A \rightarrow A_{l+1, m}^{\bullet}$ & $k_{p B A}$ \\
& $B_{l, m}^{\bullet}+B \rightarrow B_{l, m+1}^{\bullet}$ & $k_{p B B}$ \\
Termination & $A_{l, m}^{\bullet}+A_{q, r}^{\bullet} \rightarrow P_{l+q, m+r}$ or $P_{l, m}+P_{q, r}$ & $k_{t A A}$ \\
& $A_{l, m}^{\bullet}+B_{q, r}^{\bullet} \rightarrow P_{l+q, m+r}$ or $P_{l, m}+P_{q, r}$ & $k_{t A B}$ \\
& $B_{l, m}^{\bullet}+B_{q, r}^{\bullet} \rightarrow P_{l+q, m+r}$ or $P_{l, m}+P_{q, r}$ & $k_{t B B}$ \\
\hline
\end{tabular}

Table 4: Variable definitions for the copolymerisation case study.

\begin{tabular}{|c|c|}
\hline Variable & Definition \\
\hline$R_{I}$ & $2 \eta k_{d} c_{I_{2}}$ \\
\hline$k_{p A}^{*}$ & $k_{p A A} p_{A}+k_{p B A} p_{B}$ \\
\hline$k_{p B}^{*}$ & $k_{p B B} p_{B}+k_{p A B} p_{A}$ \\
\hline$p_{A}$ & $\frac{k_{p B A} c_{A}}{k_{p A B} c_{B}+k_{p B A} c_{A}}$ \\
\hline$p_{B}$ & $\frac{k_{p A B} c_{B}}{k_{p A B} c_{B}+k_{p B A} c_{A}}$ \\
\hline$k_{t}^{*}$ & $k_{t A A} p_{A}^{2}+2 k_{t A B} p_{A} p_{B}+k_{t B B} p_{B}^{2}$ \\
\hline$\tilde{k}_{d}$ & $\left(k_{p A B} k_{p B A} \frac{n_{A, 0}}{n_{B, 0}}+k_{p B B} k_{p A B}\right) \sqrt{\frac{R_{I}}{k_{t A A} k_{p B A}^{2}\left(\frac{n_{A, 0}}{n_{R} 0}\right)^{2}+2 k_{t A B} k_{p A B} k_{p B A} \frac{n_{A, 0}}{n_{B} 0}+k_{t B B} k_{p A B}^{2}}}$ \\
\hline$\dot{N}_{A, 0}$ & $n_{A 0}\left(k_{p A A} k_{p B A} \frac{n_{A 0}}{n_{B 0}}+k_{p A B} k_{p B A}\left(1-\frac{n_{A 0}}{n_{B 0}}\right)-k_{p B B} k_{p A B}\right)$. \\
\hline & $\cdot \sqrt{\frac{R_{I}}{k_{t A A} k_{p B A}^{2}\left(\frac{n_{A, 0}}{n_{B, 0}}\right)^{2}+2 k_{t A B} k_{p A B} k_{p B A} \frac{n_{A, 0}}{n_{B, 0}}+k_{t B B} k_{p A B}^{2}}}$ \\
\hline
\end{tabular}

fraction in the monomeric mixture, $X_{A}$ :

$$
\begin{aligned}
\phi_{A} & =\frac{\left(r_{A}-1\right) X_{A}^{2}+X_{A}}{\left(r_{A}-2\right) X_{A}^{2}+2 X_{A}+r_{B}\left(1-X_{A}\right)^{2}} \\
X_{A} & =\frac{n_{A}}{n_{A}+n_{B}}
\end{aligned}
$$

under the long-chain approximation. In these equations $r_{i}=\frac{k_{p i i}}{k_{p i j}}$ is the reactivity ratio of species $i$. From the $\phi_{A}$ value, using the known values of the monomer molecular weights, one can easily compute the moles of $A$ and $B$ required to produce a given mass of copolymer, $n_{A}^{\text {tot }}$ and $n_{B}^{\text {tot }}$. Since all the amount of $B$ is initially loaded into the SBR, from the $X_{A}$ value also the moles of $A$ to be initially loaded, $n_{A, 0}$, can be easily computed. The moles of $A$ to be fed to the SBR, $n_{A}^{\text {tot }}-n_{A, 0}$, can be related to the feed flowrate as 
Table 5: Process data for copolymerisation reaction.

\begin{tabular}{llc}
\hline & $A$ & \multicolumn{1}{c}{$B$} \\
\hline$M W_{i}\left[\mathrm{~kg} \mathrm{kmol}^{-1}\right]$ & 50 & \multicolumn{1}{c}{80} \\
$\rho\left[\mathrm{kg} \mathrm{m}^{-3}\right]$ & \multicolumn{2}{c}{900} \\
$F_{i}^{w}$ & 0.4 & 0.6 \\
$k_{p i i}\left[\mathrm{~m}^{3} \mathrm{kmol}^{-1} \mathrm{~s}^{-1}\right]$ & $3 \times 10^{3}$ & $1 \times 10^{3}$ \\
$r_{i}$ & 3 & 0.83 \\
$k_{t}\left[\mathrm{~m}^{3} \mathrm{kmol}^{-1} \mathrm{~s}^{-1}\right]$ & \multicolumn{2}{c}{$1 \times 10^{6}$} \\
$\eta k_{d}\left[\mathrm{~s}^{-1}\right]$ & \multicolumn{2}{c}{$1 \times 10^{-4}$} \\
\hline
\end{tabular}

follows:

$$
n_{A}^{t o t}-n_{A, 0}=\int_{0}^{t_{p r o c}} \dot{N}_{A}(t) d t=\int_{0}^{t_{p r o c}} \dot{N}_{A, 0} e^{-\tilde{k}_{d} t} d t
$$

This equation allows computing the overall process time:

$$
t_{\text {proc }}=-\frac{1}{\tilde{k}_{d}} \ln \left(1-\left(n_{A}^{\text {tot }}-n_{A, 0}\right) \frac{\tilde{k}_{d}}{\dot{N}_{A 0}}\right)
$$

This case study was worked out using the process data reported in Table 5 for a SBR aimed at producing $1 \mathrm{t}$ of copolymer. The initiator has initial concentration of $0.5 \mathrm{~mol} \%$.

Since the required value of $\phi_{A}$ is equal to 0.516 , the molar fraction of monomer $A$ in the reacting mixture, $X_{A}$, has to be equal to 0.357 from which the initial monomer amounts are readily evaluated: $n_{A 0}=4.167 \mathrm{kmol}, n_{B 0}=7.500 \mathrm{kmol}$, and $m_{0}=808.33 \mathrm{~kg}$. Therefore, using the parameter values in Table 4 , $\tilde{k}_{d}=3.604 \times 10^{-3} \mathrm{~s}^{-1}$ and $\dot{N}_{A 0}=13.82 \mathrm{~mol} \mathrm{~s}^{-1}$ are evaluated, leading to $t_{\text {proc }}=5200 \mathrm{~s}$. This process time is quite large because it reflects the continuously decreasing polymerisation rate when very high conversion is required. On the other hand, the required addition rate becomes negligible much before and the process can be interrupted much earlier without affecting product amount and quality. About $99 \%$ of the overall amount of monomer $A$ has been already injected into the reactor after slightly more than $1000 \mathrm{~s}$. This specific value has been set as the process duration, i.e. $t_{\text {proc }}=1074 \mathrm{~s}$.

\subsubsection{Transformation to continuous process}

For the sake of example, the tubular reactor is assumed to have an inlet total flowrate equal to $1 \mathrm{~kg} \mathrm{~s}^{-1}$ and a diameter of $0.3 \mathrm{~m}$. Assuming again constant density and cross sectional area, the transformation to a side-fed tubular reactor carried out using the previously discussed procedure leads to an analytical solution, summarised in the following:

1. assuming constant cross sectional area, $\hat{\mathscr{A}}=1$ follows; 
2. $\hat{\rho}^{*}(\hat{t}), \hat{F}_{i}(\hat{t})$ and $\hat{F}(\hat{t})$ are computed using Equations (24), (29) and (34), leading to:

$$
\begin{gathered}
\hat{\rho}=1 \\
\hat{F}(\hat{t})=\hat{F}_{A}(\hat{t})=a e^{-b \hat{t}} \quad \hat{F}_{B}=\hat{F}_{C}=\hat{F}_{D}=0
\end{gathered}
$$

where $a \stackrel{\text { def }}{=} \frac{\dot{N}_{A, 0} M W_{A} t_{\text {proc }}}{m_{0}}=0.918$ and $b \stackrel{\text { def }}{=} \tilde{k}_{d} t_{\text {proc }}=3.869$.

3. $\hat{Q}(\hat{\theta})$ is found from Equation (33):

$$
\hat{Q}(\hat{\theta})=1+\frac{a}{b}\left(1-e^{-b \hat{\theta}}\right)
$$

4. $K$ is computed from Equation (37):

$$
K=1+\frac{a}{b}+\frac{a}{b^{2}}\left(e^{-b}-1\right)=1.177
$$

5. the ODE (26) is solved to give a relationship between $\hat{\theta}$ and $\hat{x}$ :

$$
K \hat{x}=\left(1+\frac{a}{b}\right) \hat{\theta}+\frac{a}{b^{2}}\left(e^{-b \hat{\theta}}-1\right)
$$

6. $\hat{f}_{A}(\hat{\theta})$ is computed using Equation (38):

$$
\hat{f}_{A}(\hat{\theta})=\frac{K a b e^{-b \hat{\theta}}}{b+a\left(1-e^{-b \hat{\theta}}\right)}
$$

7. the tubular reactor length is computed using Equation (28):

$$
L=\frac{K t_{p r o c} Q_{0}}{\rho_{0} \mathscr{A}_{0}}=19.9 \mathrm{~m}
$$

8. $f_{A}(x)$ is computed using Equation (38) leading to the side feed profile shown in Figure 9.

From the numerical simulation of the side-fed tubular reactor using Equations (7) and (9), it can be easily verified that a constant value of $X_{A}=0.357$ is maintained throughout the entire reactor length: this means that the produced polymer will have the desired constant value of monomer ratio as in the SBR process.

Finally, it is worth mentioning again that kinetic information (i.e., $\dot{N}_{A 0}$ and $\tilde{k}_{d}$ ) is used only to derive the 


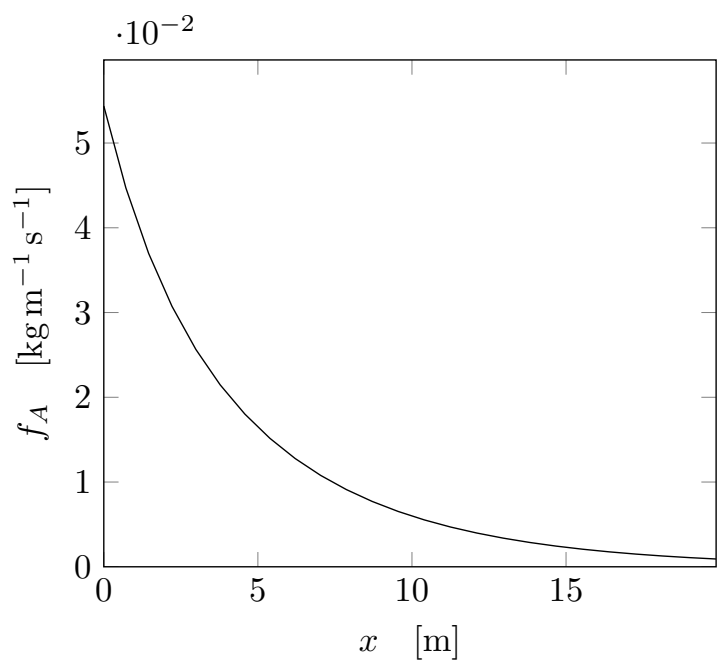

Figure 9: Side feed policy as a function of the axial coordinate for the copolymerisation reaction.

SBR feed policy. However, given the SBR feed policy (that is, given the value of $F_{i}(t)$ ), the transformation of the industrial SBR process into a tubular reactor with side injections can be carried out without any kinetic information.

\subsection{Fine chemicals}

This case study is inspired to the production of a diol, $D$, via catalytic reduction (catalyst, $C$ ) and hydrolysis (water, $W$ ) of the corresponding internal ester, $E$, as in the last step of the regioselective reaction discussed by Bringmann et al. [20]:

$$
\begin{aligned}
E+C & \rightarrow E C \\
r_{1} & =3 \times 10^{8} \exp \left(-\frac{7800}{T}\right) c_{E} c_{C} \\
E C+W & \rightarrow D+C \\
r_{2} & =1 \times 10^{8} \exp \left(-\frac{6800}{T}\right) c_{E C} c_{W}
\end{aligned}
$$

with $r\left[\mathrm{kmol} \mathrm{m}^{-3} \mathrm{~s}^{-1}\right], T[\mathrm{~K}]$ and $c_{i}\left[\mathrm{kmol} \mathrm{m}^{-3}\right] . E C$ is an intermediate complex and the reaction is carried out in a solvent, $S$.

The recipe developed at laboratory scale for the production of $D$ in a SBR is summarised in Table 6 . The reactor is initially charged with $10 \mathrm{~g} \mathrm{~S}$. Temperature is kept constant at $30^{\circ} \mathrm{C}$ throughout the entire process. 
Table 6: Laboratory scale recipe for the asymmetric synthesis.

\begin{tabular}{cl}
\hline Time $[\mathrm{s}]$ & Description \\
\hline $0-3600$ & Constant simultaneous feeding of $19.8 \mathrm{~g} 9 \mathrm{wt} \%$ solution \\
& of $E$ in $S$ and $18.36 \mathrm{~g} 2 \mathrm{wt} \%$ solution of $C$ in $S$. \\
$3600-3660$ & Idle. \\
$3660-3720$ & Constant feeding of $0.60 \mathrm{~g} \mathrm{W.}$ \\
$3720-4020$ & Idle. \\
\hline
\end{tabular}

Table 7: Process data for the diol synthesis.

\begin{tabular}{ll}
\hline$M W_{E}$ & $260 \mathrm{~kg} \mathrm{kmol}^{-1}$ \\
$M W_{C}$ & $80 \mathrm{~kg} \mathrm{kmol}^{-1}$ \\
$M W_{W}$ & $18 \mathrm{~kg} \mathrm{kmol}^{-1}$ \\
$\rho$ & $911 \mathrm{~kg} \mathrm{~m}^{-3}$ \\
$Q_{0}$ & $0.3 \mathrm{~kg} \mathrm{~s}^{-1}$ \\
$\mathscr{A}$ & $0.0314 \mathrm{~m}^{2}$ \\
\hline
\end{tabular}

Using the process data summarised in Table 7, the transformation of this SBR recipe to the one suitable for a single side-fed tubular reactor was performed through the previously discussed procedure, which in this case does not allow an analytical solution. The reactor length results equal to about $130 \mathrm{~m}$ with the feed policy summarised in Figure 10 for each species to be fed. Once more, to highlight the relevance of the use of variable feed flowrates, mass balance equations (7) and (9) were solved for both the rigorous solution and a simplified case, which assumes constant side feed flowrates. The latter were obtained through the intuitive relationships previously discussed in section 3. Since the SBR process involves four steps, the constant flowrates are computed for each tubular reactor region separately using the following relations:

$$
\begin{gathered}
\frac{f^{(k)} L^{(k)}}{Q_{0}^{(k)}}=\frac{F^{(k)} t^{(k)}}{m_{0}^{(k)}} \\
\frac{\rho \mathscr{A} L^{(k)}}{Q_{0}^{(k)}+\frac{f^{(k)} L^{(k)}}{2}}=t^{(k)}
\end{gathered}
$$

where $k=1, \ldots, 4$ specifies the considered SBR step or reactor portion. In this case, the total reactor length is the sum of the lengths of the four regions, which is equal to about $125 \mathrm{~m}$, and the side feed flowrate values for each chemical species are easily computed from the relations:

$$
\frac{f_{i}^{(k)}}{f^{(k)}}=\frac{F_{i}^{(k)}}{F^{(k)}}
$$

The results of this transformation are summarised in Figure 10 as dashed lines. In spite of the similarity 

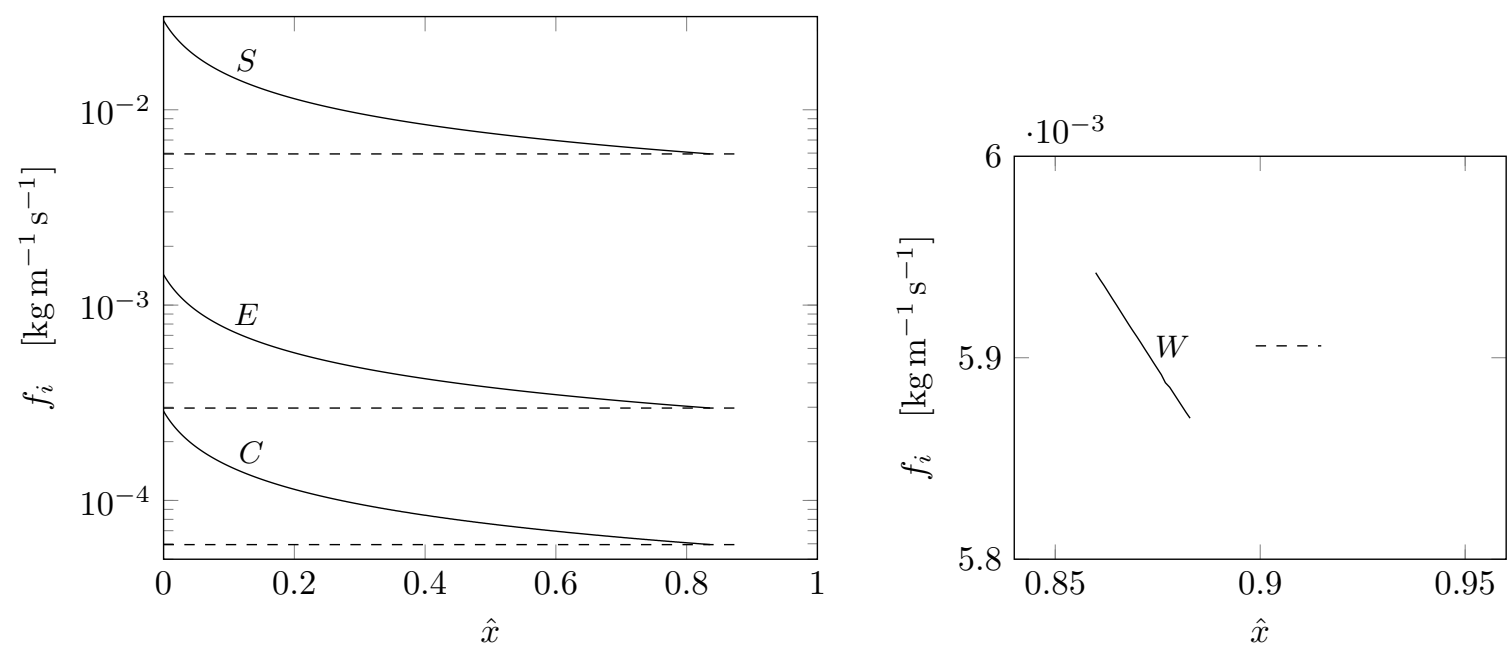

Figure 10: Side feed flowrate along the dimensionless axial coordinate according to the rigorous (solid line) and the constant feed (dashed line) method for the diol synthesis. Species $S, E$ and $C$ on the left, $W$ on the right (enlargement).

between these two side feed policies, they lead to quite different results in terms of tubular reactor performances: at constant side feed flowrates, about $40 \%$ less productivity is established than implementing the rigorous side feed flowrates. Furthermore, the final conversion is also reduced, by about $5 \%$. This means that the product quality from the constant feed tubular reactor is no more guaranteed to be the same as in the SBR plant. These results agree with the conclusions of the parametric analysis: since the rigorous solution is the only one that guarantees overlapping SBR and tubular reactor species profiles, the two reactor configurations show the same performances only in this case.

\section{Conclusions}

An approach for the transformation of isothermal homogeneous semi-batch processes into continuous ones was proposed and verified in the case of tubular reactors with distributed side injections. The key advantage of the proposed approach is that it does not require any kinetic information once the SBR recipe and some density measurements on an existing industrial plant are available. This procedure is optimal since it allows the exact reproduction of the original SBR performances. Of course, when available, kinetic information allow optimizing the reaction recipe whatever is the reactor type. However, since our primary focus is the transfer of a previously assessed recipe from SBR to the continuous tubular reactor, giving up the kinetic information becomes a major benefit.

Two case studies were analysed, a copolymer synthesis and the production of a fine chemical. In both cases, the tubular reactor ensures the same product composition and final conversion of the semi-batch 
process. Moreover, the reaction performances when applying these side feed policies have been compared with those achieved when using simpler and more intuitive feed policies, showing relevant differences.

Finally, it is worth mentioning that this tubular reactor enables scaling of productivity either changing the inlet flowrate or the cross sectional area. Being these two variables related to each other by well known design constraints, they act as a degree of freedom for the process design.

\section{Acknowledgements}

Financial support of Innovhub for one of us (FF) is gratefully acknowledged.

\section{Nomenclature}

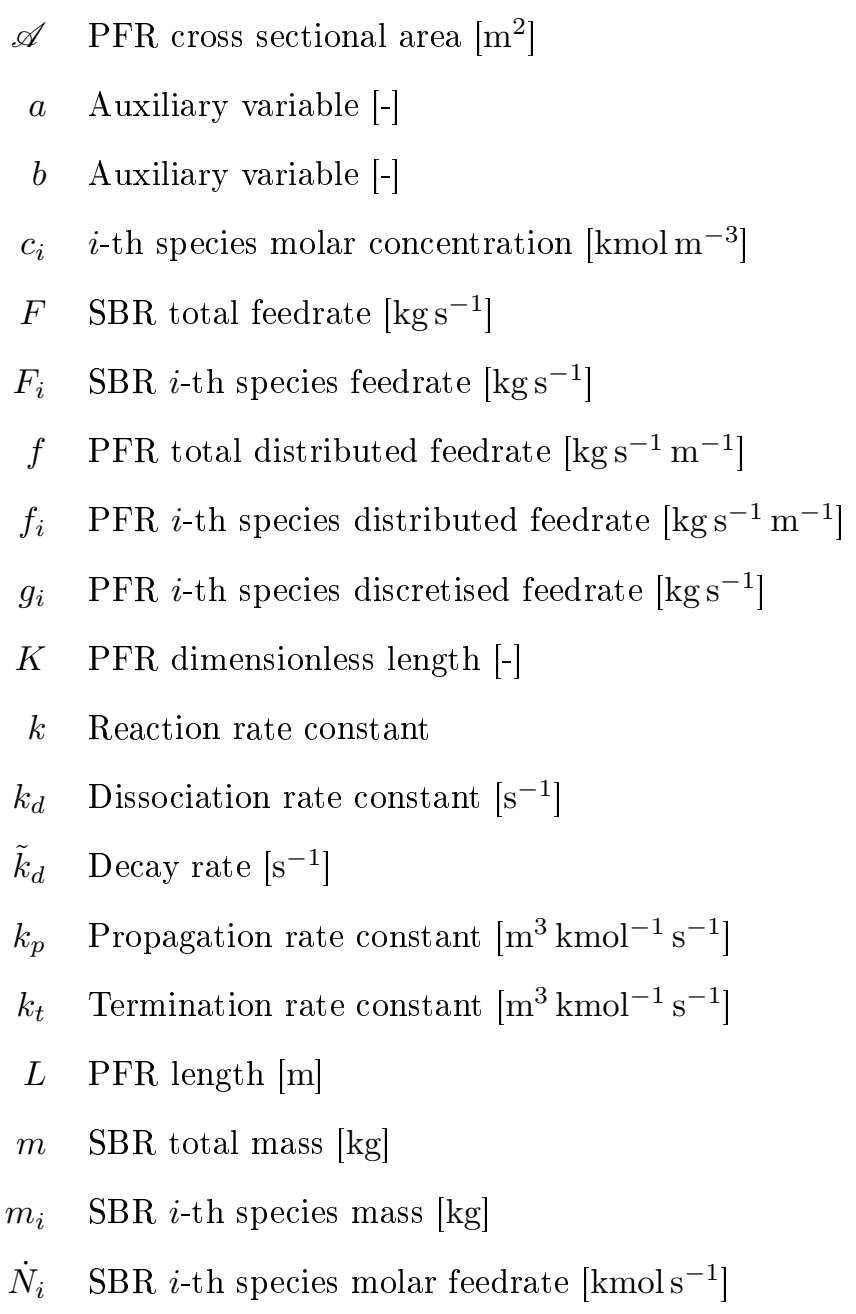


$n_{i} \quad$ SBR $i$-th species molar quantity [kmol]

$M W_{i} \quad i$-th species molecular weight $\left[\mathrm{kg} \mathrm{kmol}^{-1}\right]$

$N C \quad$ Number of components (species) [-]

$Q \quad$ PFR total flowrate $\left[\mathrm{kg} \mathrm{s}^{-1}\right]$

$Q_{i} \quad$ PFR $i$-th species flowrate $\left[\mathrm{kg} \mathrm{s}^{-1}\right]$

$R_{i} \quad i$-th species molar production rate $\left[\mathrm{kmol} \mathrm{m}^{-3} \mathrm{~s}^{-1}\right]$

$r \quad$ Molar reaction rate $\left[\mathrm{kmol} \mathrm{m}^{-3} \mathrm{~s}^{-1}\right]$

$r_{i} \quad i$-th species reactivity ratio [-]

$T \quad$ Temperature $[\mathrm{K}]$

$t \quad$ Time $[\mathrm{s}]$

$t_{\text {proc }}$ Overall process time [s]

$V \quad$ SBR volume $\left[\mathrm{m}^{3}\right]$

v Velocity $\left[\mathrm{m} \mathrm{s}^{-1}\right]$

$W_{i} \quad i$-th species overall mass $[\mathrm{kg}]$

$X_{i} \quad i$-th species monomeric molar fraction [-]

$x \quad$ PFR axial coordinate $[\mathrm{m}]$

\section{Greek symbols}

$\delta \quad$ Monomer fraction to be fed [-]

$\hat{\gamma}_{i} \quad$ Auxiliary variable [-]

$\phi_{i} \quad i$-th species desired molar fraction in copolymer [-]

$\phi_{i}^{w} \quad i$-th species desired mass fraction in copolymer [-]

$\dot{\Omega}_{i} \quad i$-th species massive production rate $\left[\mathrm{kg} \mathrm{m}^{-3} \mathrm{~s}^{-1}\right]$

$\omega_{i} \quad$ PFR $i$-th species mass fraction [-]

$\omega_{i}^{*} \quad$ SBR $i$-th species mass fraction [-]

$\rho$ Density in the PFR $\left[\mathrm{kg} \mathrm{m}^{-3}\right]$

$\rho^{*} \quad$ Density in th SBR $\left[\mathrm{kg} \mathrm{m}^{-3}\right]$

$\tau$ Residence time [s] 


\section{$\theta \quad$ Elapsed time $[\mathrm{s}]$}

\section{Other symbols}

$0 \quad$ Initial value (subscript)

(k) Diol synthesis reactor section (superscript)

$\hat{\imath}$ Dimensionless quantity .

\section{References}

[1] F. Maestri, R. Rota, Kinetic-free safe optimization of a semibatch runaway reaction: the nitration of 4-chloro benzotrifluoride, Ind. Eng. Chem. Res. 55 (2016) 12786-12794.

[2] S. Copelli, M. Derudi, J. Sempere, E. Serra, A. Lunghi, C. Pasturenzi, R. Rota, Emulsion polymerization of vinyl acetate: Safe optimization of a hazardous complex process, J. Hazard. Mater. 192 (2011) 8-17.

[3] Z. Anxionnaz, M. Cabassud, C. Gourdon, P. Tochon, Transposition of an exothermic reaction from a batch reactor to an intensified continuous one, Heat Transfer Eng. 31 (9) (2010) 788-797.

[4] C. H. Phillips, G. Lauschke, H. Peerhossaini, Intensification of batch chemical processes by using integrated chemical reactor-heat exchangers, Appl. Therm. Eng. 17 (8-10) (1997) 809-824.

[5] R. J. Minari, L. M. Gugliotta, J. R. Vega, G. R. Meira, Continuous emulsion copolymerization of acrylonitrile and butadiene. computer simulation study for improving the rubber quality and increasing production, Comput. Chem. Eng. 31 (2007) 1073-1080.

[6] S. Ferrouillat, P. Tochon, D. Della Valle, H. Peerhossaini, Open loop thermal control of exothermal chemical reactions in multifunctional heat exchangers, Int. J. Heat Mass Transfer 49 (2006) 2479-2490.

[7] K. Rossow, P. Bröge, F. G. Lüth, P. Joy, A. Mhamdi, A. Mitsos, H.-U. Moritz, W. Pauer, Transfer of emulsion polymerization of styrene and n-butyl acrylate from semi-batch to continuous tubular reactor, Macromol. React. Eng. 10 (2016) $324-338$.

[8] D. Kohlmann, M.-C. Chevrel, S. Hoppe, D. Meimaroglou, D. Chapron, P. Bourson, C. Schwede, W. Loth, A. Stammer, J. Wilson, P. Ferlin, L. Falk, S. Engell, A. Durand, Modular, flexible, and continuous plant for radical polymerization in aqueous solution, Macromol. React. Eng. 10 (2016) 339-353.

[9] T. Goerke, D. Kohlmann, S. Engell, Transfer of semibatch processes to continuous processes with side injections - opportunities and limitations, Macromol. React. Eng. 10 (2016) 364-388.

[10] D. Meimaroglou, M.-C. Chevrel, S. Hoppe, A. Durand, L. Falk, J. Wilson, P. Ferlin, On the modeling of acrylic acid copolymerization in an aqueous solution: A modular, integrated approach, Macromol. React. Eng. 10 (2016) 389-405.

[11] R. Hashemi, D. Kohlmann, S. Engell, Optimizing control and state estimation of a continuous polymerization process in a tubular reactor with multiple side-streams, Macromol. React. Eng. 10 (2016) 415-434.

[12] J. Haber, B. Jiang, T. Maeder, A. Renken, L. Kiwi-Minsker, Multi-injection microstructured reactor for intensification of fast exothermic reactions: proof of concept, Green Process. Synth. 2 (2013) 435-449.

[13] Z. Anxionnaz, M. Cabassud, C. Gourdon, P. Tochon, Heat exchanger/reactors (hex reactors): Concepts, technologies: State-of-the-art, Chem. Eng. Process. 47 (2008) 2029-2050.

[14] T. N. Zwietering, The degree of mixing in continuous flow systems, Chem. Eng. Sci. 11 (1) (1959) 1-15. 
[15] D. R. Bassett, K. L. Hoy, Nonuniform Emulsion Polymers, ACS Publications, 1981, Ch. 23 , pp. $371-387$.

[16] J. C. De La Cal, A. Echevarria, G. R. Meira, J. M. Asua, Minimum-time strategy to produce nonuniform emulsion copolymers. i. theory, J. Appl. Polym. Sci. 57 (9) (1995) 1063-1074.

[17] G. R. Gavalas, The long chain approximation in free radical reaction systems, Chem. Eng. Sci. 21 (2) (1966) $133-142$.

[18] H. Tobita, A. E. Hamielec, Kinetics of free-radical copolymerization: the pseudo-kinetic rate constant method, Polymer 32 (14) (1991) 2641-2647.

[19] F. R. Mayo, F. M. Lewis, Copolymerization. I. A basis for comparing the behavior of monomers in copolymerization; the copolymerization of styrene and methyl methacrylate, J. Am. Chem. Soc. 66 (9) (1944) $1594-1601$.

[20] G. Bringmann, M. Breuning, P. Henschel, J. Hinrichs, Asymmetric synthesis of (M)-2-hydroxymethyl-1-(2-hydroxy-4,6dimethylphenyl)naphthalene via a configurationally unstable biaryl lactone, Org. Synth. 79 (2002) 72. 
LaTeX Source Files
Click here to download LaTeX Source Files: Latex files.zip

LaTeX Source Files
Click here to download LaTeX Source Files: Latex files.zip

Click here to download LaTeX Source Files: Latex files.zip

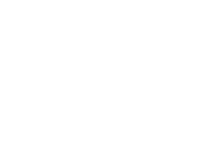

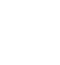

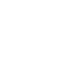

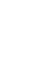
(1) (1) (1) (1) (1) (1) (1) (1) . . . . . . . . . . . .

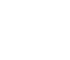

